MATHEMATICS OF COMPUTATION

Volume 74, Number 250, Pages 967-999

S 0025-5718(04)01726-0

Article electronically published on October 27, 2004

\title{
TAME KERNELS OF CUBIC CYCLIC FIELDS
}

\author{
JERZY BROWKIN
}

\begin{abstract}
There are many results describing the structure of the tame kernels of algebraic number fields and relating them to the class numbers of appropriate fields. In the present paper we give some explicit results on tame kernels of cubic cyclic fields. Table 1 collects the results of computations of the structure of the tame kernel for all cubic fields with only one ramified prime $p, 7 \leq p<5,000$.

In particular, we investigate the structure of the 7-primary and 13-primary parts of the tame kernels. The theoretical tools we develop, based on reflection theorems and singular primary units, enable the determination of the structure even of 7-primary and 13-primary parts of the tame kernels for all fields as above. The results are given in Tables 2 and 3.
\end{abstract}

\section{INTRODUCTION}

The tame kernels of number fields have been investigated by many authors (see, e.g., $\mathrm{Br} 1],[\mathrm{Br} 2,[\mathrm{CH}],[\mathrm{Gr}],[\mathrm{HK}],[\mathrm{Ke},[\mathrm{Ko} 1-[\mathrm{Ko} 4, \mathrm{Q} 1-\mathrm{Q} 4, \mathrm{Ta}$, and the references therein). In the present paper we give some explicit results for cubic cyclic fields with exactly one ramified prime. Let $F$ be a cubic cyclic field. Basic information on such fields is given, e.g., in [Ha. We assume, for simplicity, that in $F$ there is only one ramified prime $p, p>7$. Then $p \equiv 1(\bmod 6)$ and $F$ is the unique cubic subfield of the cyclotomic field $\mathbb{Q}\left(\zeta_{p}\right)$.

We investigate the tame kernel $K_{2} \mathcal{O}_{F}$, where $\mathcal{O}_{F}$ is the ring of integers of $F$.

The order of the group $K_{2} \mathcal{O}_{F}$ is easy to compute using appropriate generalized Bernoulli numbers. First, we discuss the order $\# K_{2} \mathcal{O}_{F}$ and its divisibility by small primes. To get information on the structure of the group $K_{2} \mathcal{O}_{F}$ we investigate its $q$-rank for some prime numbers $q$. For this purpose we develop an appropriate machinery (relations between tame kernels, class groups and groups of singular primary units of appropriate subfields of $F\left(\zeta_{q}\right)$ ).

We attach tables containing the results of numerical computations of tame kernels and class groups of fields $F$ in question. Our table corrects some data given in Table 1 in $[\mathrm{KK}]$. The computations have been performed using the GP/PARI package, version 2.1.0, and therefore some of the results are guaranteed to be correct only under the assumption of GRH.

Acknowledgments. A preliminary version of this paper was presented at the French-German meeting " $K$-Theory of Number Fields, from Theory to Algorithms"

Received by the editor October 17, 2002 and, in revised form, May 4, 2004.

2000 Mathematics Subject Classification. Primary 11R70; Secondary 11R16, 11Y40, 19-04, $19 \mathrm{C} 99$.

Key words and phrases. Cubic fields, cyclic fields, tame kernel. 
at the University of Metz, June 2002. I express my thanks to the organizers for the invitation and hospitality. I thank Karim Belabas for his advice on the use of GP/PARI. I am indebted to W. Narkiewicz for informing me about the paper Ma].

\section{ORDERS OF TAME KERNELS}

2.1. Basic information on the field $F$. Let $p>7, p \equiv 1(\bmod 6)$ be a prime number. Let $F$ be the unique cubic subfield of the cyclotomic field $\mathbb{Q}\left(\zeta_{p}\right)$. C.F. Gauss described the field $F$ as follows (see, e.g., [ST]). Let $g$ be a primitive root modulo $p$. Then the subgroup $H=\left\langle g^{3}\right\rangle$ of the group $(\mathbb{Z} / p)^{*}=\langle g\rangle$ has index 3 , and there are two nontrivial cosets $g H$ and $g^{2} H$. Let

$$
\alpha_{1}=\sum_{j \in H} \zeta_{p}^{j}, \quad \alpha_{2}=\sum_{j \in g H} \zeta_{p}^{j}, \quad \alpha_{3}=\sum_{j \in g^{2} H} \zeta_{p}^{j}
$$

be the Gauss periods. Then $F=\mathbb{Q}\left(\alpha_{j}\right), j=1,2,3, \alpha_{1}, \alpha_{2}, \alpha_{3}$ are conjugate in $F$, and the discriminant is $p^{2}$.

It is known that there are unique $A, B \in \mathbb{Z}$ such that

$$
4 p=A^{2}+27 B^{2}, \quad A \equiv 1 \quad(\bmod 3), \quad B>0 .
$$

In the next lemma we collect basic information on $p$ and $A$.

Lemma 2.1. If $A, B$ are as in (2.1), then

(i) $A+p+1 \equiv 0(\bmod 9)$

(ii) $A+p+1 \equiv(p-1)^{2}(\bmod 27)$,

(iii) $p(A+3) \equiv 1(\bmod 27)$.

Proof. This follows easily from $(2.1)$ and $p \equiv 1(\bmod 6)$.

The minimal polynomial for $\alpha_{j}$ is

$$
f(X)=X^{3}+X^{2}-\frac{p-1}{3} X-\frac{p(A+3)-1}{27} \in \mathbb{Z}[X] .
$$
field:

Substituting $X \mapsto \frac{1}{3}(X-1)$ we get another polynomial with the same splitting

$$
g(X)=X^{3}-3 p X-A p,
$$

with roots $\beta_{j}:=3 \alpha_{j}+1, j=1,2,3$, and discriminant $\Delta_{g}=27^{2} B^{2} p^{2}$.

Now we state some known facts on the class group of the field $F$. Let $T=$ $\operatorname{Gal}(F / \mathbb{Q})$, and let $\tau$ be a generator of $T$ satisfying $\tau\left(\zeta_{p}\right)=\zeta_{p}^{g}$.

Theorem 2.2. For a prime number $q$ let $A_{q}$ be the Sylow q-subgroup of the class group $\mathrm{Cl}\left(\mathcal{O}_{F}\right)$ of $\mathrm{F}$. Then

(i) $A_{3}=1$.

(ii) If $q \equiv 2(\bmod 3)$, then $A_{q}=B_{q} \times \tau\left(B_{q}\right)$, for some subgroup $B_{q}$ of $A_{q}$.

The same holds if we replace $\mathcal{O}_{F}$ by the ring $\mathcal{O}_{F, 2}=\mathcal{O}_{F}\left[\frac{1}{2}\right]$ of integers of $F$ localized at 2 .

Proof. (i) and (ii) follow from [Wa, Theorems $10.4(b)$, and 10.8]. The proof of Theorem 10.8 in Wa can be easily adapted to get the last statement. 
2.2. Orders of tame kernels. For every abelian totally real field $F$ the order of the group $K_{2} \mathcal{O}_{F}$ is known (see [MW] and [Wi]):

$$
\# K_{2} \mathcal{O}_{F}=w_{2}(F)\left|\zeta_{F}(-1)\right|,
$$

where $\zeta_{F}$ is the Dedekind zeta function of the field $F$, and $w_{2}(F)$ is the maximal order of a root of unity belonging to the compositum of all quadratic extensions of F.

For every cubic cyclic field $F$ we have $w_{2}(F)=24$, with two exceptions:

$$
\begin{array}{llll}
w_{2}(F)=3 \cdot 24 & \text { for } & F=\mathbb{Q}\left(\zeta_{9}\right)^{+}, \\
w_{2}(F)=7 \cdot 24 & \text { for } & F=\mathbb{Q}\left(\zeta_{7}\right)^{+},
\end{array}
$$

where $\zeta_{n}=\exp (2 \pi i / n)$ is a primitive $n$-th root of unity, and $E^{+}$denotes the maximal real subfield of a number field $E$.

Now, let us return to cubic cyclic fields $F$ with only one ramified prime $p>7$. There are at least two ways to compute $\zeta_{F}(-1)$.

1) The Dedekind zeta function $\zeta_{F}(s)$ of the field $F$ can be defined by the Euler product

$$
\zeta_{F}(s)=\left(1-\frac{1}{p^{s}}\right)^{-1} \prod_{q \text { splits }}\left(1-\frac{1}{q^{s}}\right)^{-3} \prod_{q \text { is inert }}\left(1-\frac{1}{q^{3 s}}\right)^{-1} .
$$

By the functional equation we get

$$
\zeta_{F}(-1)=-\left(\frac{p}{2 \pi^{2}}\right)^{3} \zeta_{F}(2)
$$

Consequently,

$$
\# K_{2} \mathcal{O}_{F}=\frac{3 p^{3}}{\pi^{6}} \zeta_{F}(2)
$$

From (2.3) it follows that $1<\zeta_{F}(2)<\zeta(2)^{3}$, where $\zeta(s)$ is the Riemann zeta function; hence

$$
\frac{3}{\pi^{6}} p^{3}<\# K_{2} \mathcal{O}_{F}<\frac{1}{72} p^{3}
$$

or, numerically,

$$
0.003120 p^{3}<\# K_{2} \mathcal{O}_{F}<0.01389 p^{3} .
$$

2) Now we describe the second method. The generalized Bernoulli number $B_{n, \chi}$ corresponding to a Dirichlet character $\chi$ of conductor $f$ is defined by

$$
B_{n, \chi}=f^{n-1} \sum_{j=1}^{f} \chi(j) B_{n}\left(\frac{j}{f}\right),
$$

where $B_{n}(X)$ is the $n$-th Bernoulli polynomial. Hence for the complex conjugate character $\bar{\chi}$ we have $B_{n, \bar{\chi}}=\bar{B}_{n, \chi}$.

The Dedekind zeta function of an abelian number field $F$ is the product of $L$ series:

$$
\zeta_{F}(s)=\prod_{\chi} L(s, \chi),
$$

where $\chi$ runs over the linear characters of the Galois group $\operatorname{Gal}(F / \mathbb{Q})$. 
In our case there are two nontrivial cubic Dirichlet characters $\chi$ and $\bar{\chi}$, where

$$
\chi(a)= \begin{cases}\zeta_{3}^{k}, & \text { if } a \quad(\bmod p) \in g^{k} H, k=0,1,2, \\ 0, & \text { if } p \mid a .\end{cases}
$$

Hence

$$
\zeta_{F}(s)=\zeta(s) L(s, \chi) L(s, \bar{\chi}) .
$$

Applying the formula (see Wa, Theorem 4.2])

$$
L(-1, \chi)=-\frac{B_{2, \chi}}{2}
$$

and $\zeta(-1)=-\frac{1}{12}$ we get

$$
\zeta_{F}(-1)=\zeta(-1) \frac{B_{2, \chi} \cdot B_{2, \bar{\chi}}}{4}=-\frac{1}{48}\left|B_{2, \chi}\right|^{2} .
$$

Hence

$$
\# K_{2} \mathcal{O}_{F}=\frac{1}{2}\left|B_{2, \chi}\right|^{2}
$$

It is easy to compute $B_{2, \chi}$ (see [Wa, Exercise 4.2 (a)]):

$$
B_{2, \chi}=\frac{1}{p} \sum_{j=1}^{p-1} \chi(j) j^{2} .
$$

For $k=0,1,2$, define $T_{k}:=\left\{j: 1 \leq j \leq p-1, j(\bmod p) \in g^{k} H\right\}$ and

$$
S_{k}:=\frac{1}{p} \sum_{j \in T_{k}} j^{2} .
$$

Since $j \in T_{k}$ iff $j \equiv g^{3 r+k}(\bmod p)$ for some $r, 0 \leq r<(p-1) / 3$, we get

$$
\sum_{j \in T_{k}} j^{2} \equiv g^{2 k} \sum_{r=0}^{(p-4) / 3} g^{6 r}=g^{2 k} \frac{g^{2(p-1)}-1}{g^{6}-1} \equiv 0 \quad(\bmod p),
$$

so the $S_{k}$ are integers. Moreover,

$$
S_{0}+S_{1}+S_{2}=\frac{1}{p} \sum_{j=1}^{p-1} j^{2}=\frac{p-1}{6} \cdot(2 p-1) .
$$

Now,

$$
B_{2, \chi}=\frac{1}{p} \sum_{j=1}^{p-1} \chi(j) j^{2}=S_{0}+\zeta_{3} S_{1}+\zeta_{3}^{2} S_{2}=S_{0}-\frac{1}{2}\left(S_{1}+S_{2}\right)+\frac{\sqrt{3}}{2} i\left(S_{1}-S_{2}\right) .
$$

Consequently,

$$
\begin{aligned}
\# K_{2} \mathcal{O}_{F} & =\frac{1}{2}\left|B_{2, \chi}\right|^{2}=\frac{1}{2}\left(\left(S_{0}-\frac{1}{2}\left(S_{1}+S_{2}\right)\right)^{2}+\frac{3}{4}\left(S_{1}-S_{2}\right)^{2}\right) \\
& =\frac{1}{4}\left(\left(S_{0}-S_{1}\right)^{2}+\left(S_{1}-S_{2}\right)^{2}+\left(S_{2}-S_{0}\right)^{2}\right) \\
& =\frac{1}{2}\left(\left(S_{0}+S_{1}+S_{2}\right)^{2}-3\left(S_{0} S_{1}+S_{1} S_{2}+S_{2} S_{0}\right)\right) \\
& =\frac{1}{2}\left(\left(\frac{p-1}{6} \cdot(2 p-1)\right)^{2}-3\left(S_{0} S_{1}+S_{1} S_{2}+S_{2} S_{0}\right)\right) .
\end{aligned}
$$




\subsection{Results on the order of $K_{2} \mathcal{O}_{F}$.}

\section{Theorem 2.3.}

$$
\lim _{p \rightarrow \infty} \# K_{2} \mathcal{O}_{F}=\infty
$$

Proof. This follows from (2.4).

Theorem 2.4. Let $v_{p}(m)$ be the $p$-adic valuation of $m$. Then

(i) $v_{2}\left(\# K_{2} \mathcal{O}_{F}\right)$ is odd.

(ii) For every prime number $q \equiv-1(\bmod 6)$, the number $v_{q}\left(\# K_{2} \mathcal{O}_{F}\right)$ is even. Moreover, $q \mid \# K_{2} \mathcal{O}_{F}$ iff $S_{0} \equiv S_{1} \equiv S_{2}(\bmod q)$.

(iii) $3 \mid \# K_{2} \mathcal{O}_{F}$ iff $p \equiv 1(\bmod 18)$.

(iv) If $p \equiv 1(\bmod 18)$, then

$$
3 \| \# K_{2} \mathcal{O}_{F} \quad \text { iff } \quad S_{1}, S_{2}, S_{3} \quad \text { are distinct modulo } 3 .
$$

In this case we have $\# K_{2} \mathcal{O}_{F} \equiv 6(\bmod 9)$. Moreover,

$$
3^{2} \mid \# K_{2} \mathcal{O}_{F} \quad \text { iff } \quad S_{0} \equiv S_{1} \equiv S_{2} \quad(\bmod 3) .
$$

Proof. In (2.5) put $U=S_{0}-S_{1}, V=S_{1}-S_{2}$; then $-U-V=S_{2}-S_{0}$. Consequently,

$$
\# K_{2} \mathcal{O}_{F}=\frac{1}{2}\left(U^{2}+U V+V^{2}\right) \text {. }
$$

For every prime number $q \equiv 2(\bmod 3)$ and $U, V \in \mathbb{Z}$ we have $q \mid U^{2}+U V+V^{2}$ iff $q \mid U$ and $q \mid V$. Hence $v_{q}\left(U^{2}+U V+V^{2}\right)$ is even. Then from (2.6) we get $(i)$ and (ii).

To prove $(i i i)$ we apply the last formula of $(2.5)$. From $p \equiv 1(\bmod 6)$, it follows that $2 p-1 \equiv 1 \not \equiv 0(\bmod 3)$. Hence $3 \mid \# K_{2} \mathcal{O}_{F}$ iff $3 \mid \frac{p-1}{6}$, i.e., iff $p \equiv 1(\bmod 18)$.

For $U, V \in \mathbb{Z}$ we have $3 \| U^{2}+U V+V^{2}$ iff $U \equiv V \not \equiv 0(\bmod 3)$, and $9 \mid U^{2}+U V+V^{2}$ iff $U \equiv V \equiv 0(\bmod 3)$. Moreover, if $U \equiv V \equiv \pm 1(\bmod 3)$, then $U^{2}+U V+V^{2} \equiv 3$ $(\bmod 9)$.

Therefore (iv) follows from (2.6).

Remark. Part (iii) of Theorem 2.4 follows also from a more general result of M. Kolster and A. Movahhedi ([KM], Example 2 on pp. 51-52), since a prime $p \equiv 1$ $(\bmod 6)$ is inert in the cyclotomic $\mathbb{Z}_{3}$-extension of $\mathbb{Q}$ iff $p \not \equiv 1(\bmod 18)$.

\section{THE 2-PRIMARY PART OF THE TAME KERNEL}

3.1. The 2-rank of $K_{2} \mathcal{O}_{F}$. We apply the general formula for the 2-rank of $K_{2} \mathcal{O}_{F}$, for an arbitrary number field $F$ (see [Ta]):

$$
2 \text {-rank } K_{2} \mathcal{O}_{F}=r_{1}+g_{2}-1+2-\operatorname{rank} C l\left(\mathcal{O}_{F, 2}\right),
$$

where $r_{1}$ (resp. $g_{2}$ ) is the number of real (resp. dyadic) places of $F$.

For a cubic cyclic field $F$ we have

$$
g_{2}= \begin{cases}1, & \text { if } 2 \text { is inert in } F \\ 3, & \text { if } 2 \text { splits in } F\end{cases}
$$

Then the formula (3.1) takes the form

$$
\text { 2-rank } K_{2} \mathcal{O}_{F}=2-\operatorname{rank} C l\left(\mathcal{O}_{F, 2}\right)+ \begin{cases}3, & \text { if } 2 \text { is inert in } F, \\ 5, & \text { if } 2 \text { splits in } F\end{cases}
$$


The following lemma gives conditions equivalent to the splitting of 2 in the cubic cyclic field $F$ defined in Section 2.1.

Lemma 3.1. The following conditions are equivalent:

(i) 2 splits in $F$,

(ii) $A$ is even,

(iii) $p=X^{2}+27 Y^{2}$ for some $X, Y \in \mathbb{Z}$,

(iv) 2 is a cubic residue modulo $p$.

Proof. The polynomial defined by $(2.2)$ satisfies $g(X) \equiv X^{3}+X+A(\bmod 2)$. Hence by the Hensel lemma $g(X)$ splits in $\mathbb{Q}_{2}[X]$ iff $A$ is even iff 2 splits in $F$.

The equivalence of (ii) and (iii) is obvious; for the equivalence of (iii) and (iv) see [IR, Proposition 9.6.2].

Lemma 3.2. We have

$$
\text { 2-rank } K_{2} \mathcal{O}_{F}=2-\operatorname{rank} C l\left(\mathcal{O}_{F, 2}\right)+ \begin{cases}3, & \text { if } A \text { is odd, } \\ 5, & \text { if } A \text { is even. }\end{cases}
$$

Moreover, the 2-rank of $K_{2} \mathcal{O}_{F}$ is odd.

Proof. The first assertion follows from (3.2) and Lemma 3.1. To prove the second it is sufficient to apply Theorem 2.2 to (3.2).

Lemma 3.3. Let $W_{2}(F)$ be the wild kernel of the field $F$. Then

$$
K_{2} \mathcal{O}_{F} / W_{2}(F)=(\mathbb{Z} / 2)^{r},
$$

where

$$
r= \begin{cases}3, & \text { if } 2 \text { is inert in } F, \\ 5, & \text { if } 2 \text { splits in } F .\end{cases}
$$

Proof. This follows from the description of the group $K_{2} \mathcal{O}_{F} / W_{2}(F)$ given in $\mathrm{Br} 1$, Theorem 2 .

From the above lemmas we get immediately

Corollary 3.4. For every cubic cyclic field $F$ as above we have

$$
\text { 2-rank } K_{2} \mathcal{O}_{F}=2 \text {-rank } C l\left(\mathcal{O}_{F, 2}\right)+2 \text {-rank }\left(K_{2} \mathcal{O}_{F} / W_{2}(F)\right) .
$$

3.2. Elements of order 2 in $K_{2} \mathcal{O}_{F}$. Elements of order 2 in $K_{2} \mathcal{O}_{F}$ can be determined explicitly as follows. By a theorem of Tate ([Ta, Theorem 6.3]), the group $\Delta_{F}:=\left\{a \in F^{*}:\{-1, a\}=1\right\}$ satisfies $2-\operatorname{rank}\left(\Delta_{F} / F^{* 2}\right)=1$; hence $\Delta_{F}=$ $F^{* 2} \cup 2 F^{* 2}$.

Let $\varepsilon_{1}, \varepsilon_{2}$ be fundamental units of $F$. Changing sign, if necessary, we may assume that $N \varepsilon_{1}=1$ and $\varepsilon_{2}=\tau\left(\varepsilon_{1}\right)$, where $\tau$ is a generator of the Galois group $T=$ $\operatorname{Gal}(F / \mathbb{Q})$. Then $\{-1,-1\},\left\{-1, \varepsilon_{1}\right\},\left\{-1, \tau\left(\varepsilon_{1}\right)\right\} \in K_{2} \mathcal{O}_{F}$.

Additional elements of order 2 in $K_{2} \mathcal{O}_{F}$ can be determined as follows. In view of the last statement of Theorem 2.2 we can take independent generators of the group ${ }_{2} \mathrm{Cl}\left(\mathcal{O}_{F, 2}\right)$ of the form $C l\left(\mathfrak{p}_{j}\right), C l\left(\tau\left(\mathfrak{p}_{j}\right)\right), j=1, \ldots, t$, where $2 t=2$-rank $C l\left(\mathcal{O}_{F, 2}\right)$, and $\mathfrak{p}_{j}$ are prime ideals satisfying $\mathfrak{p}_{j} \nmid 2$. Then the ideals $\mathfrak{p}_{j}^{2}$ are principal, $\mathfrak{p}_{j}^{2}=\left(\gamma_{j}\right)$, for $j=1, \ldots, t$. We may assume that $N \gamma_{j}>0$. Then $N \gamma_{j}=N \mathfrak{p}_{j}^{2}=\left(N \mathfrak{p}_{j}\right)^{2} \in F^{* 2}$.

It follows that $\left\{-1, \gamma_{j}\right\},\left\{-1, \tau\left(\gamma_{j}\right)\right\} \in K_{2} \mathcal{O}_{F}$ for $j=1, \ldots, t$. 
Finally, if 2 splits in $F,(2)=\mathfrak{p} \cdot \tau(\mathfrak{p}) \cdot \tau^{2}(\mathfrak{p})$, and the class $C l(\mathfrak{p})$ in $C l\left(\mathcal{O}_{F}\right)$ has order $r$, then the ideal $\mathfrak{p}^{r}$ is principal, $\mathfrak{p}^{r}=(\gamma)$ and $N \gamma=N\left(\mathfrak{p}^{r}\right)=2^{r}$. Consequently, $\{-1, \gamma\},\{-1, \tau(\gamma)\} \in K_{2} \mathcal{O}_{F}$.

Consider the elements

$$
-1, \varepsilon_{1}, \tau\left(\varepsilon_{1}\right), \gamma_{1}, \tau\left(\gamma_{1}\right), \ldots, \gamma_{t}, \tau\left(\gamma_{t}\right)
$$

if 2 is inert in $F$, and

$$
-1, \varepsilon_{1}, \tau\left(\varepsilon_{1}\right), \gamma, \tau(\gamma), \gamma_{1}, \tau\left(\gamma_{1}\right), \ldots, \gamma_{t}, \tau\left(\gamma_{t}\right)
$$

if 2 splits in $F$. Then the elements (3.3), respectively (3.4), are multiplicatively independent modulo $\Delta_{F}=F^{* 2} \cup 2 F^{* 2}$. It follows that the elements

$$
\{-1,-1\},\left\{-1, \varepsilon_{1}\right\},\left\{-1, \tau\left(\varepsilon_{1}\right)\right\},\left\{-1, \gamma_{j}\right\},\left\{-1, \tau\left(\gamma_{j}\right)\right\},
$$

where $j=1, \ldots, t$, are independent generators of the group ${ }_{2} K_{2} \mathcal{O}_{F}$ if 2 is inert in $F$. Similarly, the same elements and $\{-1, \gamma\},\{-1, \tau(\gamma)\}$ are independent generators of ${ }_{2} K_{2} \mathcal{O}_{F}$ if 2 splits in $F$.

We have

$$
\text { 2-rank } C l\left(\mathcal{O}_{F}\right)=2-\operatorname{rank} C l\left(\mathcal{O}_{F, 2}\right),
$$

unless 2 splits in $F,(2)=\mathfrak{p} \cdot \tau(\mathfrak{p}) \cdot \tau^{2}(\mathfrak{p})$, and the class $C l(\mathfrak{p})$ generates in $C l\left(\mathcal{O}_{F}\right)$ a direct summand of an even order.

Hence 2-rank $C l\left(\mathcal{O}_{F, 2}\right)=2$-rank $C l\left(\mathcal{O}_{F}\right)-2$ in the latter case, since in view of Theorem 2.2 both 2-ranks are even.

Theorem 3.5. For $r \geq 2$ the $2^{r}$-rank of $K_{2} \mathcal{O}_{F}$ is even.

Proof. Let $c \in K_{2} \mathcal{O}_{F}$ be an element of order $2^{r}, r \geq 2$. Then $b:=c^{2^{r-1}}$ is a nontrivial element of ${ }_{2} K_{2} \mathcal{O}_{F}$. Consequently, $b=\{-1, a\}$, where $a$ is the product of some elements (3.3), respectively (3.4),

$$
a=(-1)^{\alpha_{0}} \cdot \varepsilon_{1}^{\alpha_{1}} \cdot \tau\left(\varepsilon_{1}\right)^{\alpha_{1}^{\prime}} \cdot \gamma^{\alpha_{2}} \cdot \tau(\gamma)^{\alpha_{2}^{\prime}} \cdot \prod_{j=1}^{t} \gamma_{j}^{\beta_{j}} \cdot \tau\left(\gamma_{j}\right)^{\beta_{j}^{\prime}},
$$

where the exponents $\alpha_{0}, \alpha_{1}, \alpha_{1}^{\prime}, \alpha_{2}, \alpha_{2}^{\prime}, \beta_{j}, \beta_{j}^{\prime}$ are 0 or 1 .

Then also $\tau(c) \in K_{2} \mathcal{O}_{F}$ has order $2^{r}$. It is sufficient to prove that $\tau(b) \neq b$, or equivalently, that $\tau(a)$ and $a$ are multiplicatively independent modulo $\Delta_{F}$.

We have proved above that the norms $N \varepsilon_{1}, N \gamma, N \gamma_{j}$ belong to $\Delta_{F}$. Consequently, $\tau^{2}(\xi) \equiv(\xi \cdot \tau(\xi))^{-1}\left(\bmod \Delta_{F}\right)$, where $\xi=\varepsilon_{1}, \gamma$ or $\gamma_{j}$. Hence from (3.5) we get

$$
\begin{gathered}
\tau(a) \equiv(-1)^{\alpha_{0}} \cdot \tau\left(\varepsilon_{1}\right)^{\alpha_{1}} \cdot\left(\varepsilon_{1} \tau\left(\varepsilon_{1}\right)\right)^{-\alpha_{1}^{\prime}} \cdot \tau(\gamma)^{\alpha_{2}} \cdot(\gamma \tau(\gamma))^{-\alpha_{2}^{\prime}} \\
\cdot \prod_{j=1}^{t}\left(\tau\left(\gamma_{j}\right)^{\beta_{j}} \cdot\left(\gamma_{j} \tau\left(\gamma_{j}\right)\right)^{-\beta_{j}^{\prime}}\right) \quad\left(\bmod \Delta_{F}\right) .
\end{gathered}
$$

Assume that $\tau(a) \equiv a\left(\bmod \Delta_{F}\right)$. In view of the multiplicative independence of the elements (3.3) and (3.4) modulo $\Delta_{F}$, they should appear in (3.5) and in (3.6) with exponents of the same parity.

Therefore,

$$
\alpha_{1} \equiv-\alpha_{1}^{\prime} \quad(\bmod 2), \quad \alpha_{1}^{\prime} \equiv \alpha_{1}-\alpha_{1}^{\prime} \quad(\bmod 2) ;
$$


hence $\alpha_{1} \equiv \alpha_{1}^{\prime} \equiv 0(\bmod 2)$. In a similar way we get $\alpha_{2} \equiv \alpha_{2}^{\prime} \equiv 0(\bmod 2)$, $\beta_{j} \equiv \beta_{j}^{\prime} \equiv 0(\bmod 2)$, i.e., $a \in \Delta_{F}, b=1$. We have a contradiction since $b$ is an element of order 2 .

Corollary 3.6. We have

$$
\text { 4-rank } K_{2} \mathcal{O}_{F} \leq 2 \text {-rank } K_{2} \mathcal{O}_{F}-1 \text {, }
$$

and if an element $\varepsilon_{1}, \gamma$ or some $\gamma_{j}$ in (3.3), respectively in (3.4), is not totally positive, then

$$
\text { 4-rank } K_{2} \mathcal{O}_{F} \leq 2-\operatorname{rank} K_{2} \mathcal{O}_{F}-3 \text {. }
$$

Proof. The first inequality follows from the fact that 4-rank $K_{2} \mathcal{O}_{F} \leq 2$-rank $K_{2} \mathcal{O}_{F}$ and the two numbers are of different parities.

If an element $\alpha \in F^{*}$ is not totally positive, then applying the three real Hilbert symbols of $F$ to $\{-1, \alpha\}$ we see that $\{-1, \alpha\}$ is not a square in $K_{2} F$. In particular, $\{-1,-1\}$ is not a square. It follows that if $\varepsilon_{1}, \gamma$ or some $\gamma_{j}$ is not totally positive, then 4-rank $K_{2} \mathcal{O}_{F} \leq 2$-rank $K_{2} \mathcal{O}_{F}-2$, and the corollary follows since the two ranks are of different parities.

\section{The $q$-RANK Of The tame Kernel For $q$ ODD}

4.1. Notation. Let $q$ be an odd prime number, $\zeta_{q}$ a primitive $q$-th root of unity, and $G:=\operatorname{Gal}\left(\mathbb{Q}\left(\zeta_{q}\right) / \mathbb{Q}\right)$. Then

$$
G=\left\{\sigma_{a}: 1 \leq a \leq q-1\right\},
$$

where $\sigma_{a}\left(\zeta_{q}\right)=\zeta_{q}^{a}$. The mapping $(\mathbb{Z} / q)^{*} \longrightarrow G, a \mapsto \sigma_{a}$ is an isomorphism. For a fixed primitive root $h$ modulo $q$ the automorphism $\sigma:=\sigma_{h}$ generates $G$.

Let $\omega$ be the $q$-adic Teichmüller character of the group $(\mathbb{Z} / q)^{*}$. Then, for $1 \leq a \leq$ $q-1$, the value $\omega(a) \in \mathbb{Z}_{q}^{*}$ is determined uniquely by the conditions $\omega(a)^{q-1}=1$ and $\omega(a) \equiv a(\bmod q)$. All irreducible characters of $G=(\mathbb{Z} / q)^{*}$ are $\omega^{j}, 0 \leq j \leq q-2$. The corresponding primitive idempotents of the group ring $\mathbb{Z}_{q}[G]$ are

$$
\varepsilon_{j}=\frac{1}{q-1} \sum_{a=1}^{q-1} \omega(a)^{j} \sigma_{a}^{-1}=\frac{1}{q-1} \sum_{k=0}^{q-2} \omega(h)^{k j} \sigma^{-k}, \quad 0 \leq j \leq q-2 .
$$

In particular, $\varepsilon_{0}=\frac{1}{q-1} N$, where $N=1+\sigma+\sigma^{2}+\cdots+\sigma^{q-2}=N_{\mathbb{Q}\left(\zeta_{q}\right) / \mathbb{Q}}$ is the norm element in the group ring $\mathbb{Z}_{q}[G]$.

Since $\sigma_{a} \varepsilon_{j}=\omega(a)^{j} \varepsilon_{j}=\varepsilon_{j} \sigma_{a}$ in $\mathbb{Z}_{q}[G]$, for a $\mathbb{Z}_{q}[G]$-module $M$ we have

$$
\varepsilon_{j} M=\left\{m \in M: \sigma_{a}(m)=\omega(a)^{j} m\right\}
$$

and we get a decomposition of $M$ into a direct sum of $\mathbb{Z}_{q}[G]$-submodules:

$$
M=\bigoplus_{j=0}^{q-2} \varepsilon_{j} M=N M \oplus \bigoplus_{j=1}^{q-2} \varepsilon_{j} M .
$$

The group $\mu_{q}$ of $q$-th roots of unity has the natural structure of a $\mathbb{Z}_{q}[G]$-module. We define the action of $G$ on $\mu_{q} \otimes M$ by

$$
(\zeta \otimes m)^{\tau}=\zeta^{\tau} \otimes m^{\tau}, \quad \text { where } \quad \zeta \in \mu_{q}, m \in M, \tau \in G .
$$

Obviously,

$$
\left(\mu_{q} \otimes M\right)^{G}=\varepsilon_{0}\left(\mu_{q} \otimes M\right) .
$$


Since $\omega(a) \equiv a(\bmod q)$, we get $\zeta^{\omega(a)}=\zeta^{a}$, so

$$
\sigma_{a}(\zeta \otimes m)=\zeta^{a} \otimes \sigma_{a}(m)=\omega(a)\left(\zeta \otimes \sigma_{a}(m)\right) .
$$

Consequently, in view of (4.1),

$$
\begin{aligned}
\varepsilon_{j+1}(\zeta \otimes m) & =\frac{1}{q-1} \sum_{a=1}^{q-1} \omega(a)^{j+1} \sigma_{a}^{-1}(\zeta \otimes m)=\frac{1}{q-1} \sum_{a=1}^{q-1} \omega(a)^{j} \sigma_{a}^{-1}\left(\zeta^{a} \otimes m\right) \\
& =\frac{1}{q-1} \sum_{a=1}^{q-1} \omega(a)^{j}\left(\zeta \otimes \sigma_{a}^{-1}(m)\right)=\zeta \otimes \varepsilon_{j} m .
\end{aligned}
$$

Hence $\varepsilon_{j+1}\left(\mu_{q} \otimes M\right)=\mu_{q} \otimes \varepsilon_{j} M$ for every $G$-module $M$. In particular,

$$
\varepsilon_{0}\left(\mu_{q} \otimes M\right)=\mu_{q} \otimes \varepsilon_{q-2} M,
$$

since $\varepsilon_{q-1}=\varepsilon_{0}$.

4.2. The $q$-rank of $K_{2} \mathcal{O}_{F}$. Let $E=F\left(\zeta_{q}\right)$, where $F$ is the cubic cyclic field defined above, and $q \neq p$. Denote by $\lambda: C l\left(\mathcal{O}_{E}\right) \longrightarrow C l\left(\mathcal{O}_{E}[1 / q]\right)$ the homomorphism of the class groups induced by the imbedding $\mathcal{O}_{E} \longrightarrow \mathcal{O}_{E}[1 / q]$, and let $A=A_{E}$ be the Sylow $q$-subgroup of $C l\left(\mathcal{O}_{E}\right)$. Then $\lambda(A)$ is the Sylow $q$-subgroup of $C l\left(\mathcal{O}_{E}[1 / q]\right)$ by the surjectivity of $\lambda$.

Since $A$ is a $q$-group on which $G=\operatorname{Gal}\left(\mathbb{Q}\left(\zeta_{q}\right) / \mathbb{Q}\right)=\operatorname{Gal}(E / F)$ acts, we have

$$
A=\bigoplus_{j=0}^{q-2} \varepsilon_{j} A .
$$

Lemma 4.1. For $j \neq 0$ the mapping $\lambda: \varepsilon_{j} A \longrightarrow \varepsilon_{j} \lambda(A)$ is an isomorphism.

Proof. Since $\lambda$ commutes with the action of $G$, we have $\lambda\left(\varepsilon_{j} A\right)=\varepsilon_{j} \lambda(A)=\varepsilon_{j} A$, i.e., $\lambda$ is surjective.

Moreover, the group $\operatorname{ker} \lambda$ is generated by the classes containing prime ideals $\mathfrak{Q}$ of $E$ that divide $q$. Since $q$ does not ramify in $F$, and prime divisors $\mathfrak{q}$ of $q$ in $F$ totally ramify in $E$, it follows that $\sigma(\mathfrak{Q})=\mathfrak{Q}$ for every $\sigma \in G$.

Consequently, ker $\lambda \cap A \subset A^{G}=\varepsilon_{0} A$. Therefore ker $\lambda \cap \varepsilon_{j} A=0$ for $j \neq 0$, and the lemma follows.

Let $S^{\prime}$ be the set of prime ideals of $F$ dividing $q$ that split completely in $E$. For cubic cyclic fields the set $S^{\prime}$ is empty as follows from the next more general lemma.

Lemma 4.2. If $F$ is any Galois extension of $\mathbb{Q}$ of odd degree $d$ and $q$ is an odd prime number, then the set $S^{\prime}$ of ideals of $F$ that divide $q$ and split completely in $E=F\left(\zeta_{q}\right)$ is empty.

Proof. By assumptions $E$ is a Galois extension of $\mathbb{Q}$. For fields $K, L$, where $\mathbb{Q} \subset$ $K \subset L \subset E$, denote by $e_{q}(L / K)$ the ramification degree in the extension $L / K$ of a prime ideal of $K$ that divides $q$. Then

$$
q-1=e_{q}\left(\mathbb{Q}\left(\zeta_{q}\right) / \mathbb{Q}\right) \mid e_{q}(E / \mathbb{Q})=e_{q}(F / \mathbb{Q}) \cdot e_{q}(E / F) .
$$

Since $e_{q}(F / \mathbb{Q}) \mid(F: \mathbb{Q})=d$, where $d$ is odd, and $2 \mid q-1$, it follows that $2 \mid e_{q}(E / F)$. Hence every prime ideal of $F$ dividing $q$ ramifies in $E$, so it cannot split completely in $E$. 
Theorem 4.3. Let $E=F\left(\zeta_{q}\right)$, where $F$ is the cubic cyclic field defined above. Then

$$
q \text {-rank } K_{2} \mathcal{O}_{F}=q \text {-rank } \varepsilon_{q-2} A_{E} .
$$

Proof. There is an exact sequence

$$
0 \longrightarrow\left(\mu_{q} \otimes C l\left(\mathcal{O}_{E}[1 / q]\right)\right)^{G} \longrightarrow K_{2} \mathcal{O}_{F} / q \longrightarrow \mu_{q}^{S^{\prime}} \longrightarrow 0
$$

(see [Ke, Theorem 5.4], and [Ge]). Therefore by (4.2), (4.3) and Lemma 4.1 we get

$$
\begin{aligned}
\left(\mu_{q} \otimes C l\left(\mathcal{O}_{E}[1 / q]\right)\right)^{G} & =\left(\mu_{q} \otimes \lambda(A)\right)^{G}=\varepsilon_{0}\left(\mu_{q} \otimes \lambda(A)\right) \\
& =\mu_{q} \otimes \varepsilon_{q-2} \lambda(A)=\mu_{q} \otimes \varepsilon_{q-2} A .
\end{aligned}
$$

Since all terms in (4.4) are elementary abelian $q$-groups, (4.5) yields

$$
q-\operatorname{rank}\left(K_{2} \mathcal{O}_{F} / q\right)=q-\operatorname{rank}\left(\mu_{q} \otimes \varepsilon_{q-2} A\right)+\left|S^{\prime}\right| .
$$

Tensoring by $\mu_{q}$ and dividing by $q$ do not change the $q$-rank, and $S^{\prime}=\emptyset$ by Lemma 4.2. Hence

$$
q \text {-rank } K_{2} \mathcal{O}_{F}=q \text {-rank }\left(\varepsilon_{q-2} A\right) .
$$

Let $E^{\prime}=\mathbb{Q}\left(\zeta_{q}\right)$, and denote by $A^{\prime}$ the Sylow $q$-subgroup of $C l\left(\mathcal{O}_{E^{\prime}}\right)=C l\left(\mathbb{Z}\left[\zeta_{q}\right]\right)$. Then $A^{\prime}=1$ iff $q$ is a regular prime.

By the theorems of Herbrand and Ribet (see IR, Chapter 15, §3]) for $q>2$ we have $\varepsilon_{q-2} A^{\prime} \neq 1$ iff $q$ divides the numerator of the Bernoulli number $B_{2}=\frac{1}{6}$. Hence $\varepsilon_{q-2} A^{\prime}=1$ for every odd prime number $q$.

Theorem 4.4. If $q \equiv-1(\bmod 6)$ is a prime, and $\varepsilon_{j} A^{\prime}=1$ for some $j, 0 \leq j \leq$ $q-2$, then the $q$-rank of $\varepsilon_{j} A$ is even. In particular, the q-rank of $\varepsilon_{q-2} A$ is even.

Proof. (cf. Wa, Proof of Theorem 10.8]). Let $\tau$ be a generator of the Galois group $T:=\operatorname{Gal}(F / \mathbb{Q})=\operatorname{Gal}\left(E / \mathbb{Q}\left(\zeta_{q}\right)\right)$. Since $q \neq p$, it follows that $\sigma$ and $\tau$ commute, and consequently $T$ acts on the group $\varepsilon_{j} A$ for $0 \leq j \leq q-2$.

If $a \in \varepsilon_{j} A$ is fixed by $\tau$, then

$$
N_{E / \mathbb{Q}\left(\zeta_{q}\right)}(a)=a \cdot a^{\tau} \cdot a^{\tau^{2}}=a^{3} \quad \text { and } \quad N_{E / \mathbb{Q}\left(\zeta_{q}\right)}\left(\varepsilon_{j} A\right) \subseteq \varepsilon_{j} A^{\prime}=1,
$$

by assumption. Hence $a^{3}=1$, and so $a=1$. Thus every nontrivial orbit in $\varepsilon_{j} A$ under the action of $T$ has three elements. The same holds for orbits contained in the maximal elementary abelian $q$-subgroup $B_{j}$ of $\varepsilon_{j} A$. We have $r_{j}:=q$-rank $B_{j}=$ $q$-rank $\varepsilon_{j} A$. Hence $\# B_{j}=q^{r_{j}} \equiv 1(\bmod 3)$. Then from $q \equiv-1(\bmod 3)$ it follows that $r_{j}$ is even.

The last assertion follows from $\varepsilon_{q-2} A^{\prime}=1$.

Corollary 4.5. If $q \equiv 5(\bmod 6)$ is a prime, then the $q$-rank of $K_{2} \mathcal{O}_{F}$ is even.

By analogy with the class group of $F$ (see Theorem $2.3(i i)$ ) we state the following conjecture.

Conjecture 4.6. If $q \equiv 5(\bmod 6)$ is a prime, then

$$
\operatorname{Syl}_{q}\left(K_{2} \mathcal{O}_{F}\right)=A^{\prime} \times \tau\left(A^{\prime}\right)
$$

for some subgroup $A^{\prime}$ of the Sylow q-subgroup of $K_{2} \mathcal{O}_{F}$. 
4.3. The case $q=3$. If $q=3$, we can give a more precise description of the fields in question. From

$$
4 p=A^{2}+27 B^{2}
$$

it follows that in the field $\mathbb{Q}\left(\zeta_{3}\right)$ the prime $p$ splits into two principal ideals, $(p)=$ $(\pi)(\bar{\pi})$, where

$$
\pi=\frac{1}{2}(A+3 B \sqrt{-3})=\frac{1}{2}(A+3 B)+3 B \zeta_{3},
$$

and $\bar{\pi}$ is the complex conjugate of $\pi$.

Moreover,

$$
E=F\left(\zeta_{3}\right)=\mathbb{Q}\left(\zeta_{3}, \Pi\right), \quad \text { where } \quad \Pi=\sqrt[3]{\bar{\pi} / \pi} .
$$

In fact, one can easily verify that

$$
\beta:=\pi \Pi+\bar{\pi} / \Pi=\sqrt[3]{p \pi}+\sqrt[3]{p \bar{\pi}} \in \mathbb{Q}\left(\zeta_{3}, \Pi\right)
$$

is a root of $g(X)=X^{3}-3 p X-A p$. Equivalently, one can use the Cardano formulas.

Hence $E=F\left(\zeta_{3}\right)=\mathbb{Q}\left(\beta, \zeta_{3}\right) \subseteq \mathbb{Q}\left(\zeta_{3}, \Pi\right)$ and (4.6) follows, since both fields in question are of degree 6 over $\mathbb{Q}$.

Theorem 4.7. We have

$$
\text { 3-rank } K_{2} \mathcal{O}_{F}=3 \text {-rank } A_{E} .
$$

Proof. For $q=3$ we have

$$
\varepsilon_{0}=\frac{1}{2}(1+\sigma)=\frac{1}{2} N, \quad \varepsilon_{1}=\frac{1}{2}(1-\sigma) .
$$

Hence $\varepsilon_{0} A_{E}=N_{E / F}\left(A_{E}\right)=1$, since the class number of $F$ is not divisible by 3 (by Theorem $2.2(i))$. Consequently, $\varepsilon_{1} A_{E}=\left(\varepsilon_{0}+\varepsilon_{1}\right) A_{E}=A_{E}$, and the assertion follows from Theorem 4.3.

Now we can give a direct proof of a result obtained earlier.

Theorem 4.8. If $p \equiv 1(\bmod 9)$, then $3-\operatorname{rank} K_{2} \mathcal{O}_{F} \geq 1$.

Proof. Set $\gamma=\sqrt[3]{p}$ for simplicity. We shall prove that $E(\gamma)$ is an abelian unramified extension of $E$ of degree 3. Then 3-rank $A_{E} \geq 1$, and the assertion follows from Theorem 4.7.

Since the extension $E / \mathbb{Q}$ is abelian, and $\gamma=\sqrt[3]{p}$ cannot belong to any abelian extension of $\mathbb{Q}$ (the Galois group of the polynomial $X^{3}-p \in \mathbb{Q}[X]$ is $S_{3}$ ), it follows that $(E(\gamma): E)=3$. Moreover, $E(\gamma)$ is a cyclic extension of $E$, since $\zeta_{3} \in E$.

Evidently $(p)=(\gamma)^{3}$ is the cube of an ideal in $\mathcal{O}_{E}$, and the congruence $X^{3} \equiv p$ $\left(\bmod \left(1-\zeta_{3}\right) 3\right)$ has a solution $X=1$ in $\mathcal{O}_{E}$, since $p \equiv 1(\bmod 9)$. Hence $p$ is a singular primary element in $E$ with respect to the prime number 3 . From Wa. Exercise $9.3(b)]$ it follows that $E(\gamma)$ is an unramified extension of $E$, as claimed.

\section{REFLECTION THEOREMS}

We apply reflection theorems to prove some estimates of the $q$-rank $K_{2} \mathcal{O}_{F}$; for more details see $\mathrm{Br} 2$. We extend the above notation as follows.

Let $L$ be the maximal unramified and elementary abelian $q$-extension of $E$ with the Galois group $H:=\operatorname{Gal}(L / E)$. Then the Artin reciprocity map gives an isomorphism of $G$-modules $A / q \longrightarrow H$. 
By Kummer theory, $L=E\left(B^{1 / q}\right)$, where $B$ is a subgroup of $E^{*}$ containing $E^{* q}$. Set $B_{0}:=B / E^{* q}$. Then every principal ideal $(b)$, where $b \in B$, is the $q$-th power of an ideal in $E$, since $L / E$ is unramified. Moreover, $B_{0}$ is isomorphic to the dual $\widehat{H}$ of $H$ as a $G$-module.

Define ${ }_{q} A=\left\{a \in A: a^{q}=1\right\}$. Then there is a homomorphism of $G$-modules

$$
\varphi: B_{0} \longrightarrow{ }_{q} A
$$

such that $\varphi\left(b E^{* q}\right)=C l(\mathfrak{a})$, where the ideal $\mathfrak{a}$ of $\mathcal{O}_{E}$ is defined by the condition $(b)=\mathfrak{a}^{q}$.

Theorem 5.1 (See Br2, Theorem 3.1]).

$$
q-\operatorname{rank}\left(\varepsilon_{j} A\right)=q-\operatorname{rank}\left(\varepsilon_{q-j} B_{0}\right) .
$$

Let $U_{E}$ be the group of units of $\mathcal{O}_{E}$, and denote by $U_{E}^{\prime}$ its subgroup of units $u$ satisfying

$$
u \equiv x^{q} \quad\left(\bmod q\left(1-\zeta_{q}\right)\right)
$$

for some $x \in O_{E}$. Such a $u$ is called a singular primary unit. It is easy to see that $U_{E}^{q} \subseteq U_{E}^{\prime}$

Theorem 5.2 (See $\mathrm{Br} 2$ (3.1)).

$$
\operatorname{ker} \varphi=U_{E}^{\prime} / U_{E}^{q}
$$

Theorem 5.3. We have

$$
q \text {-rank }\left(\varepsilon_{2}\left(U_{E}^{\prime} / U_{E}^{q}\right)\right) \leq q-\operatorname{rank}\left(K_{2} \mathcal{O}_{F}\right) \leq q-\operatorname{rank}\left(\varepsilon_{2} A_{E}\right)+q-\operatorname{rank}\left(\varepsilon_{2}\left(U_{E}^{\prime} / U_{E}^{q}\right)\right) .
$$

Proof. Since $\varphi: B_{0} \longrightarrow{ }_{q} A$ is a homomorphism of $G$-modules, it induces homomorphisms of the corresponding direct summands:

$$
\varphi_{j}: \varepsilon_{j} B_{0} \longrightarrow \varepsilon_{j}\left({ }_{q} A\right) \quad \text { for } \quad 0 \leq j \leq q-2 .
$$

Hence

$$
q-\operatorname{rank}\left(\operatorname{ker} \varphi_{j}\right) \leq q-\operatorname{rank}\left(\varepsilon_{j} B_{0}\right) \leq q-\operatorname{rank}\left(\varepsilon_{j}\left({ }_{q} A\right)\right)+q-\operatorname{rank}\left(\operatorname{ker} \varphi_{j}\right) .
$$

Since $\operatorname{ker} \varphi_{j}=\varepsilon_{j} \operatorname{ker} \varphi$ and $q-\operatorname{rank}(A)=q$-rank $\left({ }_{q} A\right)$, the assertion follows from Theorems 5.1, 5.2 for $j=2$, and 4.3.

Theorem 5.3 gives some estimates from below and from above of the $q$-rank of $K_{2} \mathcal{O}_{F}$ by means of the $q$-ranks of some subgroups of the class group and of the group of singular primary units (modulo $q$-th powers) of the field $E=F\left(\zeta_{q}\right)$.

For large prime numbers $q$, the degree $(E: \mathbb{Q})=q-1$ is large, and it is difficult to determine its class group and the group of units, and the action of the Galois $\operatorname{group} \operatorname{Gal}(E / \mathbb{Q})$ on them. We prove that sometimes $E$ can be replaced by its proper subfields, where the necessary computations are much easier to perform.

For a fixed primitive root $h$ modulo $q$ set $\omega(h)=\zeta_{q-1} \in \mathbb{Z}_{q}^{*}$, and $t=\frac{q-1}{2}$. Then $\sigma^{t}$ is the complex conjugation on $E$. The maximal real subfield $E^{+}$of $E$ is the subfield fixed by $\sigma_{t}$. Then $N_{E / E^{+}}=1+\sigma^{t}$.

Lemma 5.4. Under the above notation we have

$$
\varepsilon_{2}=\rho \cdot N_{E / E^{+}} \quad \text { for some } \rho \in \mathbb{Z}_{q}[G] .
$$


Proof. By the definition of $\varepsilon_{2}$ we get

$$
\varepsilon_{2}=\frac{1}{q-1} \sum_{k=0}^{q-2} \zeta_{q-1}^{2 k} \sigma^{-k}=\frac{1}{q-1} \sum_{k=0}^{t-1} \zeta_{t}^{k} \sigma^{-k}\left(1+\sigma^{t}\right)=\frac{1}{q-1} \sum_{k=0}^{t-1} \zeta_{t}^{k} \sigma^{-k} \cdot N_{E / E^{+}} .
$$

Assume that $q \equiv 1(\bmod 6)$, and for $j=0,1,2$, let

$$
\eta_{j}:=\frac{1}{3} \sum_{\ell=0}^{2} \zeta_{3}^{\ell j} \tau^{-\ell}
$$

where $\zeta_{3} \in \mathbb{Z}_{q}$ and $\tau$ is a generator of $T:=\operatorname{Gal}(F / \mathbb{Q})=\operatorname{Gal}\left(E / \mathbb{Q}\left(\zeta_{q}\right)\right)$. Then $\eta_{0}, \eta_{1}, \eta_{2}$ are primitive idempotents of the group ring $\mathbb{Z}_{q}[T]$. Hence

$$
\varepsilon_{2}=\sum_{j=0}^{2} \varepsilon_{2} \eta_{j}
$$

in the group ring $\mathbb{Z}_{q}[G \times T]$. Set $r=(q-1) / 6$. For $j=0,1,2$, let $T_{j}$ be the subgroup of $G \times T$ generated by $\sigma^{t}$ and $\sigma^{r j} \tau^{-1}$, and denote by $E_{j}$ the subfield of $E$ fixed by $T_{j}$. Then $\# T_{j}=6, E_{j} \subset E^{+}$, and $\left(E_{j}: \mathbb{Q}\right)=(q-1) / 2=t$ for $j=0,1,2$. In particular, $E_{0}=E^{\left\langle\sigma^{t}, \tau^{-1}\right\rangle}=\mathbb{Q}\left(\zeta_{q}\right)^{+}$is the maximal real subfield of $\mathbb{Q}\left(\zeta_{q}\right)$.

Lemma 5.5. Under the above notation we have

$$
\varepsilon_{2} \eta_{j}=\rho_{j} \cdot N_{E / E_{j}}
$$

for $j=0,1,2$ and some $\rho_{j} \in \mathbb{Z}_{q}[G \times T]$.

Proof. In the definition of $\varepsilon_{2}$ (see (4.1)) there is a summation over $k$, where $0 \leq k \leq$ $q-2$. Since $q-1=6 r$, we can substitute $k=i r+m$, where $0 \leq i \leq 5,0 \leq m \leq r-1$.

Then

$$
\begin{aligned}
\varepsilon_{2} \eta_{j} & =\frac{1}{3(q-1)} \sum_{\ell=0}^{2} \zeta_{3}^{\ell j} \tau^{-\ell} \sum_{i=0}^{5} \sum_{m=0}^{r-1} \zeta_{6 r}^{2 i r+2 m} \sigma^{-i r-m} \\
& =\frac{1}{3(q-1)}\left(\sum_{m=0}^{r-1} \zeta_{3 r}^{m} \sigma^{-m}\right) \cdot\left(\sum_{\ell=0}^{2} \sum_{i=0}^{5} \zeta_{3}^{\ell j+i} \sigma^{-i r} \tau^{-\ell}\right) .
\end{aligned}
$$

Denote the first sum in brackets by $\xi_{1}$, and the second by $\xi_{2}$. For fixed $\ell$ put $s=\ell j+i$; then for $0 \leq i \leq 5, s$ runs over all residues modulo 6 . Hence

$$
\xi_{2}=\sum_{\ell=0}^{2} \sum_{s=0}^{5} \zeta_{3}^{s} \sigma^{-r(s-\ell j)} \tau^{-\ell}=\left(\sum_{s=0}^{5} \zeta_{3}^{s} \sigma^{-r s}\right) \cdot\left(\sum_{\ell=0}^{2}\left(\sigma^{r j} \tau^{-1}\right)^{\ell}\right) .
$$

Denote the first factor of the last expression by $\xi_{3}$, and the second by $\xi_{4}$. Then, since $3 r=t$ and $\sigma^{-t}=\sigma^{t}$, we get

$\xi_{3}=1+\zeta_{3} \sigma^{-r}+\zeta_{3}^{2} \sigma^{-2 r}+\sigma^{t}+\zeta_{3} \sigma^{-r} \sigma^{t}+\zeta_{3}^{2} \sigma^{-2 r} \sigma^{t}=\xi_{5} \cdot\left(1+\sigma^{t}\right)=\xi_{5} \cdot N_{E / E^{+}}$, where $\xi_{5}=1+\zeta_{3} \sigma^{-r}+\zeta_{3}^{2} \sigma^{-2 r}$.

Similarly, for $j=0,1,2$,

$$
\xi_{4}=\sum_{\ell=0}^{2}\left(\sigma^{r j} \tau^{-1}\right)^{\ell}
$$

is the norm from $E$ to the subfield fixed by $\sigma^{r j} \tau^{-1}$. Hence $\left(1+\sigma^{t}\right) \xi_{4}$ is the norm from $E$ to the subfield of $E^{+}$fixed by $\sigma^{r j} \tau^{-1}$, i.e., $\left(1+\sigma^{t}\right) \xi_{4}=N_{E / E_{j}}$. 
Finally we get

$$
\varepsilon_{2} \eta_{j}=\xi_{1} \xi_{2}=\xi_{1} \xi_{3} \xi_{4}=\xi_{1} \xi_{5}\left(1+\sigma^{t}\right) \xi_{4}=\xi_{1} \xi_{5} N_{E / E_{j}} .
$$

For every subfield $L$ of $E$ we define $U_{L}^{\prime}$ to be the group of singular primary units in $L$,

$$
U_{L}^{\prime}=U_{E}^{\prime} \cap L
$$

Since $\operatorname{Gal}(E / \mathbb{Q})$ acts on $U_{E}^{\prime}$, it follows that

$$
N_{E / L} U_{E}^{\prime} \subseteq U_{L}^{\prime}
$$

Lemma 5.6. The natural inclusion $U_{L}^{\prime} \longrightarrow U_{E}^{\prime}$ induces an injection

$$
U_{L}^{\prime} / U_{L}^{q} \longrightarrow U_{E}^{\prime} / U_{E}^{q} \quad \text { for } \quad q>3 .
$$

Proof. Assume that $\varepsilon \in U_{L}^{\prime} \cap U_{E}^{q}$. Then $\varepsilon=\eta^{q}$ for some $\eta \in U_{E}$. Consequently, $\eta=$ $\sqrt[q]{\varepsilon}$, and so $(L(\eta): L)=1$ or $q$. On the other hand, $(L(\eta): L) \mid(E: \mathbb{Q})=3(q-1)$, which is not divisible by $q$. Hence $(L(\eta): L)=1$, i.e., $\eta \in U_{L}, \varepsilon \in U_{L}^{q}$, and the lemma follows.

From the above we get inclusions of elementary abelian $q$-groups

$$
N_{E / L}\left(U_{E}^{\prime} / U_{E}^{q}\right) \subseteq U_{L}^{\prime} / U_{L}^{q} \subseteq U_{E}^{\prime} / U_{E}^{q} ;
$$

hence, acting by $\varepsilon_{2}$, we obtain

$$
q \text {-rank }\left(\varepsilon_{2} N_{E / L}\left(U_{E}^{\prime} / U_{E}^{q}\right)\right) \leq q-\operatorname{rank}\left(\varepsilon_{2}\left(U_{L}^{\prime} / U_{L}^{q}\right)\right) \leq q-\operatorname{rank}\left(\varepsilon_{2}\left(U_{E}^{\prime} / U_{E}^{q}\right)\right) .
$$

Lemma 5.7. Let $L_{1}, L_{2}$ be subfields of $E$, and set $L_{0}=L_{1} \cap L_{2}$. Assume that $L_{1}, L_{2}$ are proper subfields of $L_{1} L_{2}$, and $U_{L_{0}}^{\prime} / U_{L_{0}}^{q}=1$. Then the mapping

$$
U_{L_{1}}^{\prime} / U_{L_{1}}^{q} \times U_{L_{2}}^{\prime} / U_{L_{2}}^{q} \quad \longrightarrow \quad U_{L_{1} L_{2}}^{\prime} / U_{L_{1} L_{2}}^{q},
$$

given by $\left(u_{1}, u_{2}\right) \mapsto u_{1} u_{2}$, is injective.

Proof. Suppose that $u_{1} \in U_{L_{1}}^{\prime}, u_{2} \in U_{L_{2}}^{\prime}$ satisfy

$$
u_{1} u_{2}=u^{q}, \quad \text { for some } u \in U_{L_{1} L_{2}} \text {. }
$$

We have

$$
\begin{gathered}
N_{L_{1} L_{2} / L_{1}}\left(u_{1}\right)=u_{1}^{r}, \quad \text { where } r=\left(L_{1} L_{2}: L_{1}\right) \mid(E: \mathbb{Q})=3(q-1), \\
N_{L_{1} L_{2} / L_{1}}\left(u_{2}\right)=N_{L_{2} / L_{0}}\left(u_{2}\right) \in U_{L_{0}}^{\prime}=U_{L_{0}}^{q} \subseteq U_{L_{1}}^{q}, \\
N_{L_{1} L_{2} / L_{1}}\left(u^{q}\right)=\left(N_{L_{1} L_{2} / L_{1}}(u)\right)^{q} \subseteq U_{L_{1}}^{q} .
\end{gathered}
$$

Consequently, from (5.3), it follows that $u_{1}^{r} \in U_{L_{1}}^{q}$, so $u_{1} \in U_{L_{1}}^{q}$, since $q \nmid r$. By symmetry we get $u_{2} \in U_{L_{2}}^{q}$; hence the mapping in question is injective.

From Lemma 5.7 it also follows that the restricted mapping

$$
\varepsilon_{2}\left(U_{L_{1}}^{\prime} / U_{L_{1}}^{q}\right) \times \varepsilon_{2}\left(U_{L_{2}}^{\prime} / U_{L_{2}}^{q}\right) \quad \longrightarrow \quad \varepsilon_{2}\left(U_{L_{1} L_{2}}^{\prime} / U_{L_{1} L_{2}}^{q}\right)
$$

is injective. Consequently, under the assumptions of Lemma 5.7 we have that

$$
\begin{aligned}
\sum_{j=1}^{2} q-\operatorname{rank}\left(\varepsilon_{2}\left(U_{L_{j}}^{\prime} / U_{L_{j}}^{q}\right)\right) & \leq q-\operatorname{rank}\left(\varepsilon_{2}\left(U_{L_{1} L_{2}}^{\prime} / U_{L_{1} L_{2}}^{q}\right)\right) \\
& \leq q-\operatorname{rank}\left(\varepsilon_{2}\left(U_{E}^{\prime} / U_{E}^{q}\right)\right) .
\end{aligned}
$$


Theorem 5.8. Let $E_{j}$ be the subfield of $E$ fixed by the group $T_{j}=\left\langle\sigma^{r j} \tau^{-1}, \sigma^{t}\right\rangle$. Then

$$
\begin{aligned}
\max _{0 \leq j \leq 2} & \left(q-\operatorname{rank}\left(\varepsilon_{2}\left(U_{E_{j}}^{\prime} / U_{E_{j}}^{q}\right)\right)\right) \leq q-\operatorname{rank}\left(K_{2} \mathcal{O}_{F}\right) \\
& \leq \sum_{j=0}^{2} q-\operatorname{rank}\left(\varepsilon_{2} A_{E_{j}}\right)+\sum_{j=0}^{2} q-\operatorname{rank}\left(\varepsilon_{2}\left(U_{E_{j}}^{\prime} / U_{E_{j}}^{q}\right)\right) .
\end{aligned}
$$

Moreover, if the class number of the field $\mathbb{Q}\left(\zeta_{q}\right)$ is not divisible by $q$, and in the field $L_{0}:=E_{1} \cap E_{2}$ we have $U_{L_{0}}^{\prime} / U_{L_{0}}^{q}=1$, then

$$
\begin{aligned}
& \sum_{j=1}^{2} q \text {-rank }\left(\varepsilon_{2}\left(U_{E_{j}}^{\prime} / U_{E_{j}}^{q}\right)\right) \leq q-\operatorname{rank}\left(K_{2} \mathcal{O}_{F}\right) \\
& \quad \leq \sum_{j=1}^{2} q-\operatorname{rank}\left(\varepsilon_{2} A_{E_{j}}\right)+\sum_{j=1}^{2} q-\operatorname{rank}\left(\varepsilon_{2}\left(U_{E_{j}}^{\prime} / U_{E_{j}}^{q}\right)\right) .
\end{aligned}
$$

Proof. The first inequality in (5.5) follows from (5.2) and Theorem 5.3.

By Lemma 5.5, from (5.1) we get

$$
\begin{aligned}
q-\operatorname{rank}\left(\varepsilon_{2} A_{E}\right) & =\sum_{j=0}^{2} q-\operatorname{rank}\left(\varepsilon_{2} \eta_{j} A_{E}\right) \leq \sum_{j=0}^{2} q-\operatorname{rank}\left(\varepsilon_{2} N_{E / E_{j}}\left(A_{E}\right)\right) \\
& \leq \sum_{j=0}^{2} q-\operatorname{rank}\left(\varepsilon_{2} A_{E_{j}}\right) .
\end{aligned}
$$

Similarly, by (5.2) we obtain

$$
q \text {-rank }\left(\varepsilon_{2}\left(U_{E}^{\prime} / U_{E}^{q}\right)\right) \leq \sum_{j=0}^{2} q-\operatorname{rank}\left(\varepsilon_{2}\left(U_{E_{j}}^{\prime} / U_{E_{j}}^{q}\right)\right) .
$$

Now the second inequality in (5.5) follows from Theorem 5.3.

Let us observe that $E_{0}$ is the field fixed by the group $\left\langle\tau^{-1}, \sigma^{t}\right\rangle$; hence $E_{0}=$ $\mathbb{Q}\left(\zeta_{q}\right)^{+}$. From the assumption $q$-rank $\left(A_{\mathbb{Q}\left(\zeta_{q}\right)}\right)=0$ it follows that $q$-rank $\left(A_{E_{0}}\right)=0$ and that there is no abelian unramified extension of $\mathbb{Q}\left(\zeta_{q}\right)$ of degree $q$. Then from Wa, Exercise 9.3 ] we deduce that every singular primary unit in $\mathbb{Q}\left(\zeta_{q}\right)$ is a $q$-th power of a unit. By Lemma 5.6 the same holds for the field $\mathbb{Q}\left(\zeta_{q}\right)^{+}$.

Thus we have proved that $q$-rank $\left(\varepsilon_{2}\left(U_{E_{0}}^{\prime} / U_{E_{0}}^{q}\right)\right)=0$.

Now we proceed as in the proof of (5.5). To prove the first inequality in (5.6) we use (5.4) with $L_{j}=E_{j}$ for $j=1,2$, and Theorem 5.3.

To prove the second inequality it is sufficient to observe that on the right-hand side of (5.5) the summands corresponding to $j=0$ vanish, as proved above.

\section{The 7-RANK OF $K_{2} \mathcal{O}_{F}$}

We apply Theorem 5.8 with $q=7$ to prove some estimates for the 7-rank of $K_{2} \mathcal{O}_{F}$. In many cases these estimates suffice to determine the structure of the 7primary part of the group $K_{2} \mathcal{O}_{F}$. Our arguments can be extended mutatis mutandis to other primes $q \equiv 1(\bmod 6)$.

We are going to give an effective description of the fields $E_{j}$ to look for singular primary units in these fields, and to describe the action of $\varepsilon_{2}$ on these units. For this purpose we have to fix some notation. 
6.1. Notation. For a fixed primitive root $g$ modulo $p$, where $p \equiv 1(\bmod 6)$ is a prime, we have defined the Gauss periods

$$
\alpha_{1}=\sum_{k=1}^{(p-1) / 3} \zeta_{p}^{g^{3 k}}, \quad \alpha_{2}=\sum_{k=1}^{(p-1) / 3} \zeta_{p}^{g^{3 k+1}}, \quad \alpha_{3}=\sum_{k=1}^{(p-1) / 3} \zeta_{p}^{g^{3 k+2}} .
$$

Obviously, $\alpha_{1}$ does not depend on the primitive root $g$ chosen, but if we replace $g$ by $g^{-1}$, then $\alpha_{2}$ and $\alpha_{3}$ permute; hence the number $\left(\alpha_{1}-\alpha_{2}\right)\left(\alpha_{2}-\alpha_{3}\right)\left(\alpha_{3}-\alpha_{1}\right)$ changes sign.

We shall assume henceforth that a primitive root $g$ modulo $p$ is chosen in such a way that

$$
\left(\alpha_{1}-\alpha_{2}\right)\left(\alpha_{2}-\alpha_{3}\right)\left(\alpha_{3}-\alpha_{1}\right)>0 .
$$

In particular, for the prime 7 , we choose the primitive root 3 . Then the Gauss periods are

$$
\gamma_{1}=\zeta_{7}+\zeta_{7}^{-1}=1.24, \quad \gamma_{2}=\zeta_{7}^{3}+\zeta_{7}^{-3}=-1.80, \quad \gamma_{3}=\zeta_{7}^{2}+\zeta_{7}^{-2}=-0.44 ;
$$

hence $\gamma_{1}>\gamma_{3}>\gamma_{2}$, and so $\left(\gamma_{1}-\gamma_{2}\right)\left(\gamma_{2}-\gamma_{3}\right)\left(\gamma_{3}-\gamma_{1}\right)>0$.

Since $\gamma_{1}, \gamma_{2}, \gamma_{3}$ are the roots of the polynomial $f(X)=X^{3}+X^{2}-2 X-1$ with discriminant

$$
\Delta_{f}=\left(\left(\gamma_{1}-\gamma_{2}\right)\left(\gamma_{2}-\gamma_{3}\right)\left(\gamma_{3}-\gamma_{1}\right)\right)^{2}=49,
$$

it follows that

$$
\left(\gamma_{1}-\gamma_{2}\right)\left(\gamma_{2}-\gamma_{3}\right)\left(\gamma_{3}-\gamma_{1}\right)=7
$$

On the other hand,

$$
\left(\gamma_{1}-\gamma_{2}\right)\left(\gamma_{2}-\gamma_{3}\right)\left(\gamma_{3}-\gamma_{1}\right)=U_{\gamma}-V_{\gamma}
$$

where

$$
U_{\gamma}=\gamma_{1} \gamma_{2}^{2}+\gamma_{2} \gamma_{3}^{2}+\gamma_{3} \gamma_{1}^{2}, \quad V_{\gamma}=\gamma_{1}^{2} \gamma_{2}+\gamma_{2}^{2} \gamma_{3}+\gamma_{3}^{2} \gamma_{1}
$$

Moreover, by the Vieta formulas,

$$
U_{\gamma}+V_{\gamma}=\sum \gamma_{1} \gamma_{2} \cdot \sum \gamma_{1}-3 \gamma_{1} \gamma_{2} \gamma_{3}=-1
$$

Solving the system $U_{\gamma}-V_{\gamma}=7, U_{\gamma}+V_{\gamma}=-1$ we get $U_{\gamma}=3, V_{\gamma}=-4$.

We denote by $\sigma$ the automorphism of the field $\mathbb{Q}\left(\zeta_{7}\right)$ satisfying $\sigma\left(\zeta_{7}\right)=\zeta_{7}^{3}$. Then $\sigma\left(\gamma_{i}\right)=\gamma_{i+1}$, where the indices are taken modulo 3 . We repeat the same for the cubic subfield $F$ of the cyclotomic field $\mathbb{Q}\left(\zeta_{p}\right)$, where $p \equiv 1(\bmod 6), p>7$. The field $F$ is generated by any root $\beta_{i}$ of the polynomial

$$
g(X)=X^{3}-3 p X-A p
$$

with discriminant $\Delta_{g}=(27 B p)^{2}$. Since $\beta_{i}=3 \alpha_{i}+1$, where $\alpha_{i}$ are the Gauss sums given above, from our assumption on the primitive root $g$ modulo $p$ it follows that

$$
\left(\beta_{1}-\beta_{2}\right)\left(\beta_{2}-\beta_{3}\right)\left(\beta_{3}-\beta_{1}\right)=27\left(\alpha_{1}-\alpha_{2}\right)\left(\alpha_{2}-\alpha_{3}\right)\left(\alpha_{3}-\alpha_{1}\right)>0 .
$$

Moreover, the automorphism $\tau \in \operatorname{Gal}\left(\mathbb{Q}\left(\zeta_{p}\right) / \mathbb{Q}\right)$ given by $\tau\left(\zeta_{p}\right)=\zeta_{p}^{g}$ satisfies $\tau\left(\beta_{i}\right)=\beta_{i+1}$, where the indices are taken modulo 3 .

As above we set

$$
U_{\beta}=\beta_{1} \beta_{2}^{2}+\beta_{2} \beta_{3}^{2}+\beta_{3} \beta_{1}^{2}, \quad V_{\beta}=\beta_{1}^{2} \beta_{2}+\beta_{2}^{2} \beta_{3}+\beta_{3}^{2} \beta_{1} .
$$

Then

$$
U_{\beta}-V_{\beta}=27 B p, \quad U_{\beta}+V_{\beta}=-3 A p
$$


hence

$$
U_{\beta}=-\frac{3}{2}(A-9 B), \quad V_{\beta}=-\frac{3}{2}(A+9 B) .
$$

Now let us consider the compositum of the cubic fields $\mathbb{Q}\left(\zeta_{7}\right)^{+}=\mathbb{Q}\left(\gamma_{i}\right)$ and $F=$ $\mathbb{Q}\left(\beta_{i}\right)$. It is the maximal real subfield $E^{+}$of $E=F\left(\zeta_{7}\right)$. Since

$$
\operatorname{Gal}\left(E^{+} / \mathbb{Q}\right)=\mathbb{Z} / 3 \times \mathbb{Z} / 3=\langle\sigma\rangle \times\langle\tau\rangle,
$$

where the automorphisms $\sigma$ and $\tau$ are restricted to $E^{+}$, there are four cubic subfields of $E^{+}$. Namely, $F=\mathbb{Q}\left(\beta_{i}\right), E_{0}=\mathbb{Q}\left(\zeta_{7}\right)^{+}=\mathbb{Q}\left(\gamma_{i}\right), E_{1}$, the subfield fixed by $\sigma \tau^{-1}$, and $E_{2}$, the subfield fixed by $\sigma \tau$.

Hence, for $a \in E^{+}$we have

$$
a \in E_{1} \quad \text { iff } \quad \sigma(a)=\tau(a), \quad \text { and } \quad a \in E_{2} \quad \text { iff } \quad \sigma(a)=\tau^{-1}(a) .
$$

Define

$$
\rho_{1}=\gamma_{1} \beta_{1}+\gamma_{2} \beta_{3}+\gamma_{3} \beta_{2}, \quad \rho_{2}=\gamma_{1} \beta_{1}+\gamma_{2} \beta_{2}+\gamma_{3} \beta_{3} .
$$

It is easy to see that $\rho_{1}, \rho_{2} \in E^{+}$, and $\sigma\left(\rho_{1}\right)=\tau\left(\rho_{1}\right), \sigma\left(\rho_{2}\right)=\tau^{-1}\left(\rho_{2}\right)$. Moreover, $\rho_{1}, \rho_{2} \notin \mathbb{Q}$. Therefore,

$$
E_{1}=\mathbb{Q}\left(\rho_{1}\right), \quad E_{2}=\mathbb{Q}\left(\rho_{2}\right) .
$$

Applying the Vieta formulas one can verify that the minimal polynomials for $\rho_{1}$ and $\rho_{2}$ are, respectively,

$$
\begin{aligned}
& g_{1}(X)=X^{3}-21 p X-7 p \frac{A-27 B}{2}, \\
& g_{2}(X)=X^{3}-21 p X-7 p \frac{A+27 B}{2},
\end{aligned}
$$

with discriminants

$$
\Delta_{g_{1}}=3^{6} \cdot 7^{2} \cdot p^{2} \cdot\left(\frac{A+B}{2}\right)^{2} \quad \text { and } \quad \Delta_{g_{2}}=3^{6} \cdot 7^{2} \cdot p^{2} \cdot\left(\frac{A-B}{2}\right)^{2} .
$$

6.2. The action of $\varepsilon_{2}$. Let a cyclic group $G=\langle\sigma\rangle$ of order 6 act on an elementary abelian 7-group $A$. In the following lemma we describe the action on $A$ of the primitive idempotents

$$
\varepsilon_{j}=\frac{1}{6} \sum_{k=0}^{5} \omega(3)^{k j} \sigma^{-k}, \quad 0 \leq j<6,
$$

of the group ring $\mathbb{Z}_{7}[G]$, where $\omega(3) \in \mathbb{Z}_{7}$ satisfies $\omega(3)^{6}=1, \omega(3) \equiv 3(\bmod 7)$.

We apply this lemma in the case when $\varepsilon_{j}=\varepsilon_{2}, G=\operatorname{Gal}\left(\mathbb{Q}\left(\zeta_{7}\right) / \mathbb{Q}\right)$, and $A$ is either the maximal elementary abelian 7-subgroup of the class group of $E_{j}$, or the group of classes of singular primary units of $E_{j}$ modulo seventh powers.

Lemma 6.1. Let a cyclic group $G=\langle\sigma\rangle$ of order 6 act on an elementary abelian 7-group $A$, and let $a \in A$.

(i) If

$$
\left(1+\sigma+\sigma^{2}\right) a=1,
$$

then $\varepsilon_{j} a=1$ for $j=0,1,3,5$.

(ii) Moreover,

$$
\begin{array}{lllll}
\sigma(a)=a^{2} & \text { iff } & \left(\varepsilon_{2} a=a\right. & \text { and } & (6.1) \text { holds }) \\
\sigma(a)=a^{4} & \text { iff } \quad\left(\varepsilon_{4} a=a\right. & \text { and } & (6.1) \text { holds }) .
\end{array}
$$


Proof. (i) Let us observe that (6.1) implies $\sigma^{3} a=a$, so

$$
\begin{aligned}
\varepsilon_{j} a & =\frac{1}{6} \sum_{k=0}^{5} 3^{k j} \sigma^{-k} \cdot a=\frac{1}{6} \sum_{k=0}^{2}\left(3^{k j}+3^{(k+3) j}\right) \sigma^{-k} \cdot a \\
& =-\left(1+3^{3 j}\right) \sum_{k=0}^{2} 3^{k j} \sigma^{-k} \cdot a .
\end{aligned}
$$

Since $3^{3 j} \equiv(-1)^{j}(\bmod 7)$, it follows that $\varepsilon_{j} a=1$ for $j$ odd. Moreover, $\varepsilon_{0} a=$ $\frac{1}{6}\left(1+\sigma+\sigma^{2}\right) a=1$, by (6.1). This proves $(i)$.

(ii) Assume that $\sigma(a)=a^{2}$. Then $\left(1+\sigma+\sigma^{2}\right) a=a \cdot a^{2} \cdot a^{4}=a^{7}=1$. Thus (6.1) holds.

Now, by $(6.2)$ and (6.1),

$$
\varepsilon_{2} a=-\left(1+3^{6}\right)\left(1+3^{2} \sigma^{-1}+3^{4} \sigma^{-2}\right) a=(2+3 \sigma) a=a^{2} \cdot \sigma(a)^{3} .
$$

Therefore, $\varepsilon_{2} a=a$ iff $a \cdot \sigma(a)^{3}=1$ iff $\sigma(a)=a^{2}$.

The proof of the last statement is similar.

6.3. Estimates of the 7-rank of $K_{2} \mathcal{O}_{F}$. The class group of the field $\mathbb{Q}\left(\zeta_{7}\right)$ is trivial, and there are no nontrivial singular primary units with respect to $q=7$ in the field $E_{1} \cap E_{2}=\mathbb{Q}$. Therefore, the assumptions of the "moreover" part of Theorem 5.8 are satisfied, and from (5.6) we get

$$
\begin{aligned}
& \sum_{j=1}^{2} 7-\operatorname{rank}\left(\varepsilon_{2}\left(U_{E_{j}}^{\prime} / U_{E_{j}}^{7}\right)\right) \leq 7-\operatorname{rank}\left(K_{2} \mathcal{O}_{F}\right) \\
& \quad \leq \sum_{j=1}^{2} 7-\operatorname{rank}\left(\varepsilon_{2} A_{E_{j}}\right)+\sum_{j=1}^{2} 7-\operatorname{rank}\left(\varepsilon_{2}\left(U_{E_{j}}^{\prime} / U_{E_{j}}^{7}\right)\right) .
\end{aligned}
$$

Lemma 6.2. Assume that the class numbers of the fields $E_{1}$ and $E_{2}$ are not divisible by 7. Then equality holds in (6.3):

$$
7-\operatorname{rank}\left(K_{2} \mathcal{O}_{F}\right)=\sum_{j=1}^{2} 7-\operatorname{rank}\left(\varepsilon_{2}\left(U_{E_{j}}^{\prime} / U_{E_{j}}^{7}\right)\right) .
$$

Proof. From the assumption it follows that $7-\operatorname{rank}\left(\varepsilon_{2} A_{E_{j}}\right)=0$ for $j=1,2$, so (6.3) yields the assertion.

6.4. Computation of the 7-rank of $K_{2} \mathcal{O}_{F}$. We give some details concerning the computation of the 7-rank of $K_{2} \mathcal{O}_{F}$ via the 7-ranks of other groups appearing in (6.3).

Since the field $E_{j}, j=1,2$, is cubic and totally real, there are two fundamental units $\xi_{1}, \xi_{2}$ in $E_{j}$, and we can assume that $\xi_{2}=\sigma\left(\xi_{1}\right)$ and $N_{E_{j} / \mathbb{Q}}\left(\xi_{1}\right)=1$. Therefore $U_{E_{j}} / U_{E_{j}}^{7}=\mathbb{F}_{7}^{2}$, and the characteristic polynomial of the action of $\sigma$ on the linear space $U_{E_{j}} / U_{E_{j}}^{7}$ over $\mathbb{F}_{7}$ is $X^{2}+X+1$. Since the polynomial has distinct roots 2,4 in $\mathbb{F}_{7}$, it follows that the corresponding eigenspaces $V_{j}^{(2)}$ and $V_{j}^{(4)}$ are 1-dimensional.

From Lemma 6.1 it follows that

$$
\varepsilon_{2}\left(U_{E_{j}}^{\prime} / U_{E_{j}}^{7}\right)=V_{j}^{(2)} \cap\left(U_{E_{j}}^{\prime} / U_{E_{j}}^{7}\right) .
$$

Consequently,

$$
7 \text {-rank }\left(\varepsilon_{2}\left(U_{E_{j}}^{\prime} / U_{E_{j}}^{7}\right)\right) \leq \operatorname{dim} V_{j}^{(2)}=1,
$$


and to determine this 7-rank it is sufficient to find a unit $u \in U_{E_{j}}$ such that the corresponding coset $u U_{E_{j}}$ is an eigenvector corresponding to the eigenvalue 2, i.e., $\sigma(u) \equiv u^{2}\left(\bmod U_{E_{j}}^{7}\right)$, and to verify whether $u \in U_{E_{j}}^{\prime}$.

This last verification can be done as follows. The field $E_{j}$ equals $\mathbb{Q}\left(\rho_{j}\right)$, where $\rho_{j}$ is a root of the polynomial $g_{j}(X)$ defined above. Let us observe that, for $p>7$,

$$
A^{2}-B^{2} \equiv A^{2}+27 B^{2}=4 p \not \equiv 0 \quad(\bmod 7) ;
$$

hence $A \not \equiv \pm B(\bmod 7)$. Consequently, $g_{j}(X)$ is an Eisenstein polynomial with respect to 7 . Hence the root $x:=\rho_{j}$ of $g_{j}(X)$ satisfies $v_{7}(x)=\frac{1}{3}$, where $v_{7}$ is the normalized 7 -adic valuation, $v_{7}(7)=1$.

Consequently, every unit $u \in U_{E_{j}}$ can be written in the form

$$
u=a_{0}+a_{1} x+a_{2} x^{2}, \quad \text { where } \quad a_{i} \in \mathbb{Q}, \quad v_{7}\left(a_{0}\right)=0 .
$$

Raising to the sixth power induces an isomorphism of the group $U_{E_{j}} / U_{E_{j}}^{7}$; hence we can replace $u$ by $u^{6}$. In that case $a_{0} \equiv 1(\bmod 7)$.

So let us assume that in (6.5) we have $a_{0} \equiv 1(\bmod 7)$. Then $u$ is a singular primary unit iff $u \equiv 1(\bmod 7 x)$, or, equivalently,

$$
a_{0} \equiv 1 \quad(\bmod 49), \quad a_{1} \equiv a_{2} \equiv 0 \quad(\bmod 7) .
$$

We proceed similarly to determine the 7-rank of $A_{E_{j}}$. We look for generators of the maximal elementary abelian 7-subgroup of the class group $A_{E_{j}}$. It is a linear space over $\mathbb{F}_{7}$. Then we describe the action of the automorphism $\sigma$ on it and determine the eigenspace $W_{j}^{(2)}$ corresponding to the eigenvalue 2. In view of Lemma 6.1,

$$
7 \text {-rank }\left(\varepsilon_{2} A_{E_{j}}\right)=\operatorname{dim} W_{j}^{(2)} \quad \text { for } \quad j=1,2 .
$$

The results of the computations are given in Table 2 (at the end of the paper), where we used the following shorthand notation, for $j=1,2$ :

$$
v_{7}:=v_{7}\left(\# K_{2} \mathcal{O}_{F}\right), d_{j}:=\operatorname{dim}\left(V_{j}^{(2)} \cap\left(U_{E_{j}}^{\prime} / U_{E_{j}}^{7}\right)\right), h_{j}:=7-\operatorname{rank}\left(\varepsilon_{2} A_{E_{j}}\right),
$$

and $\left(K_{2} \mathcal{O}_{F}\right)_{7}$ is the 7-primary part of $K_{2} \mathcal{O}_{F}$.

Thus if a coset $u U_{E_{j}}^{7}$ generates the linear space $V_{j}^{(2)}$, then $d_{j}=1$ or 0 according as $u$ is a singular primary unit or not.

It turns out that the 7-primary part $A_{E_{j}}$ of the class group of the field $E_{j}$ is nontrivial in three cases only:

- For $p=2707$ we have $A_{E_{1}}=\mathbb{Z} / 7$. We have computed that $\varepsilon_{2} A_{E_{1}}=$ 1 (fortunately!), so the class group does not contribute to the 7-rank of $K_{2} \mathcal{O}_{F}$. Consequently, by (6.4),

$$
7-\operatorname{rank}\left(K_{2} \mathcal{O}_{F}\right)=\sum_{j=1}^{2} 7-\operatorname{rank}\left(\varepsilon_{2}\left(U_{E_{j}}^{\prime} / U_{E_{j}}^{7}\right)\right)=1 .
$$

- For $p=3313$ we have $A_{E_{2}}=\mathbb{Z} / 7$. Since

$$
\sum_{j=1}^{2} 7 \text {-rank }\left(\varepsilon_{2}\left(U_{E_{j}}^{\prime} / U_{E_{j}}^{7}\right)\right)=0,
$$

it follows that $1 \leq 7-\operatorname{rank}\left(K_{2} \mathcal{O}_{F}\right) \leq 7-\operatorname{rank}\left(\varepsilon_{2} A_{E_{2}}\right) \leq 7-\operatorname{rank}\left(A_{E_{2}}\right)=1$; hence equality holds everywhere, and 7 -rank $K_{2} \mathcal{O}_{F}=1$. 
- For $p=4513$ we have $A_{E_{1}}=\mathbb{Z} / 7$. Since $7^{2} \| \# K_{2} \mathcal{O}_{F}$ and

$$
\sum_{j=1}^{2} 7-\operatorname{rank}\left(\varepsilon_{2}\left(U_{E_{j}}^{\prime} / U_{E_{j}}^{7}\right)\right)=2,
$$

it follows that 7-rank $\left(K_{2} \mathcal{O}_{F}\right)=2$, and the knowledge of the 7-rank of $\varepsilon_{2} A_{E_{1}}$ is not necessary.

\section{The 13-RANK OF $K_{2} \mathcal{O}_{F}$}

Now we apply Theorem 5.8 with $q=13$. The arguments are similar to those in the case $q=7$ considered above, so we omit some details.

For the prime number 13 we choose the primitive root 2 and we consider the Gauss periods

$$
\gamma_{j}=\zeta_{13}^{2^{j}}+\zeta_{13}^{-2^{j}}, \quad 1 \leq j \leq 6 .
$$

Then the automorphism $\sigma$ such that $\sigma\left(\zeta_{13}\right)=\zeta_{13}^{2}$ satisfies $\sigma\left(\gamma_{j}\right)=\gamma_{j+1}, 1 \leq j \leq 6$, where the indices are taken modulo 6 .

Evidently $\mathbb{Q}\left(\gamma_{j}\right)=\mathbb{Q}\left(\zeta_{13}\right)^{+}$for $1 \leq j \leq 6$. Moreover, the field $\mathbb{Q}\left(\gamma_{j}\right)$ contains a unique cubic subfield $F_{0}:=\mathbb{Q}\left(\gamma_{j}+\gamma_{j+3}\right)$ and a unique quadratic subfield $\mathbb{Q}(\sqrt{13})=\mathbb{Q}\left(\gamma_{j}+\gamma_{j+2}+\gamma_{j+4}\right)$.

Let $F$ be the cubic subfield of the cyclotomic field $\mathbb{Q}\left(\zeta_{p}\right)$, where $p \equiv 1(\bmod 6)$, $p>13$. Then $F=\mathbb{Q}\left(\beta_{i}\right)$, where $\beta_{i}, i=1,2,3$, are the roots of the polynomial

$$
g(X)=X^{3}-3 p X-A p,
$$

satisfying $\left(\beta_{1}-\beta_{2}\right)\left(\beta_{2}-\beta_{3}\right)\left(\beta_{3}-\beta_{1}\right)>0$. The automorphism $\tau$ of the field $F$ is defined by $\tau\left(\beta_{i}\right)=\beta_{i+1}$, where the indices are taken modulo 3 .

Let $E^{+}$be the maximal real subfield of the field $E=F\left(\zeta_{13}\right)$. Then $E^{+}$is the compositum of the fields $\mathbb{Q}\left(\zeta_{13}\right)^{+}$and $F$, and consequently,

$$
\operatorname{Gal}\left(E^{+} / \mathbb{Q}\right)=\mathbb{Z} / 6 \times \mathbb{Z} / 3=\langle\sigma\rangle \times\langle\tau\rangle,
$$

where the automorphisms $\sigma$ and $\tau$ are restricted to $E^{+}$.

For $j=0,1,2$, let $E_{j}$ be the subfield of $E^{+}$fixed by the automorphism $\sigma^{2 j} \tau^{-1}$. Then $E_{0}=\mathbb{Q}\left(\zeta_{13}\right)^{+}$. It follows that, for $a \in E^{+}$,

$$
\begin{array}{lll}
a \in E_{1} & \text { iff } & \sigma^{2}(a)=\tau(a), \\
a \in E_{2} & \text { iff } & \sigma^{2}(a)=\tau^{-1}(a) .
\end{array}
$$

Define

$$
\begin{aligned}
& \rho_{1}=\gamma_{1} \beta_{1}+\gamma_{5} \beta_{2}+\gamma_{3} \beta_{3}, \\
& \rho_{2}=\gamma_{1} \beta_{1}+\gamma_{3} \beta_{2}+\gamma_{5} \beta_{3} .
\end{aligned}
$$

Then $\rho_{1}, \rho_{2} \in E^{+}$and $\sigma^{2}\left(\rho_{1}\right)=\tau\left(\rho_{1}\right), \sigma^{2}\left(\rho_{2}\right)=\tau^{-1}\left(\rho_{2}\right)$. Moreover, $\sigma^{k}\left(\rho_{j}\right) \neq \rho_{j}$ for $1 \leq k \leq 5, j=1,2$. Hence $E_{j}=\mathbb{Q}\left(\rho_{j}\right)$, for $j=1,2$.

Let

$$
\begin{aligned}
& \lambda_{1}=\rho_{1}+\sigma \tau\left(\rho_{1}\right)=\left(\gamma_{1}+\gamma_{4}\right) \beta_{1}+\left(\gamma_{2}+\gamma_{5}\right) \beta_{2}+\left(\gamma_{3}+\gamma_{6}\right) \beta_{3}, \\
& \lambda_{2}=\rho_{2}+\sigma \tau^{2}\left(\rho_{2}\right)=\left(\gamma_{1}+\gamma_{4}\right) \beta_{1}+\left(\gamma_{3}+\gamma_{6}\right) \beta_{2}+\left(\gamma_{2}+\gamma_{5}\right) \beta_{3} .
\end{aligned}
$$

Then $F_{j}:=\mathbb{Q}\left(\lambda_{j}\right)$ is a cubic subfield of $E_{j}, j=1,2$.

There are ten fields $F$ corresponding to primes $p<5000$ such that $13^{2} \mid \# K_{2} \mathcal{O}_{F}$. Namely, for

$$
p=163,379,487,757,1723,1867,3673,4447,4999
$$

the above divisibility holds, and even in the stronger form $13^{2} \| \# K_{2} \mathcal{O}_{F}$. 
The class number of the field $E_{1} \cap E_{2}=\mathbb{Q}(\sqrt{13})$ is 1 , and there are no nontrivial singular primary units with respect to 13 . Consequently, for $a \in U_{E_{j}}^{\prime} / U_{E_{j}}^{13}$ we have $N_{E_{j} / \mathbb{Q}(\sqrt{13})}(a)=1$.

Similarly, if in the field $F_{j}, j=1,2$, there are no nontrivial singular primary units, $U_{F_{j}}^{\prime} / U_{F_{j}}^{13}=1$, then for $a \in U_{E_{j}}^{\prime} / U_{E_{j}}^{13}$ we have $N_{E_{j} / F_{j}}(a)=1$.

Since $\operatorname{Gal}\left(E_{j} / F_{j}\right)=\left\langle\sigma^{3}\right\rangle$ and $\operatorname{Gal}\left(E_{j} / \mathbb{Q}(\sqrt{13})\right)=\left\langle\sigma^{2}\right\rangle$, the above equalities for norms can be written as

$$
\left(1+\sigma^{3}\right) a=1 \quad \text { and } \quad\left(1+\sigma^{2}+\sigma^{4}\right) a=1 \quad \text { for } \quad a \in U_{E_{j}}^{\prime} / U_{E_{j}}^{13} .
$$

The following analogue of Lemma 6.1 holds.

Lemma 7.1. Let $A$ be an elementary abelian 13-group on which the Galois group $G=\operatorname{Gal}\left(\mathbb{Q}\left(\zeta_{13}\right) / \mathbb{Q}\right)=\langle\sigma\rangle$ acts, and let $a \in A$.

(i) If

$$
\left(1+\sigma^{2}+\sigma^{4}\right) a=1 \quad \text { and } \quad\left(1+\sigma^{3}\right) a=1,
$$

then

$$
\varepsilon_{j} a=1 \quad \text { for } \quad 0 \leq j<11, \quad j \neq 2,10 \text {. }
$$

(ii) Moreover,

$$
\begin{aligned}
& \sigma(a)=a^{4} \quad \text { iff } \quad\left(\varepsilon_{2} a=a \quad \text { and } \quad(7.1) \text { holds }\right), \\
& \sigma(a)=a^{10} \quad \text { iff } \quad\left(\varepsilon_{10} a=a \quad \text { and } \quad(7.1) \text { holds }\right) \text {. }
\end{aligned}
$$

Proof. (i) From the definition of $\varepsilon_{j}$ and (7.1) we get

$$
\begin{aligned}
\varepsilon_{j} a & =\frac{1}{12} \sum_{k=0}^{11} 2^{k j} \sigma^{-k} \cdot a \\
& =\frac{1}{12} \sum_{k=0}^{2}\left(2^{k j} \sigma^{-k}+2^{(k+3) j} \sigma^{-k-3}+2^{(k+6) j} \sigma^{-k-6}+2^{(k+9) j} \sigma^{-k-9}\right) \cdot a \\
& =\frac{1}{12}\left(1-2^{3 j}+2^{6 j}-2^{9 j}\right) \sum_{k=0}^{2} 2^{k j} \sigma^{-k} \cdot a \\
& =\frac{1}{12}\left(1-2^{3 j}\right)\left(1+2^{6 j}\right) \sum_{k=0}^{2} 2^{k j} \sigma^{-k} \cdot a .
\end{aligned}
$$

Since $2^{6 j} \equiv(-1)^{j}(\bmod 13)$, it follows that $\varepsilon_{j} a=0$ for $j$ odd and for $j$ divisible by 4 .

For $j=6$, by (7.1) we have

$$
\sum_{k=0}^{2} 2^{k j} \sigma^{-k} \cdot a=\sum_{k=0}^{2}(-1)^{k} \sigma^{-k} \cdot a=\left(1-\sigma+\sigma^{2}\right) a=\left(1+\sigma^{2}+\sigma^{4}\right) a=0 .
$$

(ii) Assume (7.1). Then, in particular, (7.2) holds for $j=2$ :

$$
\begin{aligned}
\varepsilon_{2} a & =\frac{1}{12}\left(1-2^{6}\right)\left(1+2^{12}\right) \sum_{k=0}^{2} 2^{2 k} \sigma^{-k} \cdot a \\
& =\frac{1}{3}\left(1+4 \sigma^{-1}+3 \sigma^{-2}\right) a=-4\left(1-4 \sigma^{2}-3 \sigma\right) a=(2 \sigma+6) a=\sigma(a)^{2} \cdot a^{6}
\end{aligned}
$$

in view of (7.1). Hence $\varepsilon_{2} a=a$ iff $\sigma(a)^{2} \cdot a^{5}=1$, equivalently, $\sigma(a)=a^{4}$. 
Now let $\sigma(a)=a^{4}$. Then $\left(1+\sigma^{2}+\sigma^{4}\right) a=a \cdot a^{4^{2}} \cdot a^{4^{4}}=a^{13}=1$. Similarly $\left(1+\sigma^{3}\right) a=a \cdot a^{4^{3}}=a \cdot a^{-1}=1$. Consequently, (7.1) holds, and from the above it follows that $\varepsilon_{2} a=a$.

The proof of the last equivalence is similar.

Lemma 7.2. Assume that the class numbers of the fields $E_{1}$ and $E_{2}$ are not divisible by 13 . Then

$$
13-\operatorname{rank}\left(K_{2} \mathcal{O}_{F}\right)=\sum_{j=1}^{2} 13-\operatorname{rank}\left(\varepsilon_{2}\left(U_{E_{j}}^{\prime} / U_{E_{j}}^{13}\right)\right) .
$$

Proof. This follows from (5.6).

The field $E_{j}$ is totally real of degree 6 , so there are five fundamental units in $E_{j}$. The automorphism $\sigma$ acts on the linear space $U_{E_{j}} / U_{E_{j}}^{13}=\mathbb{F}_{13}^{5}$ over $\mathbb{F}_{13}$. Denote by $V_{j}^{(\lambda)}$ the eigenspace corresponding to an eigenvalue $\lambda$. It is known (see $\underline{\mathrm{Ma}}$ ) that the characteristic polynomial of the action of $\sigma$ on this linear space is equal to $\left(X^{6}-1\right) /(X-1)$. Since the polynomial has five distinct roots in $\mathbb{F}_{13}$ :

$$
\left(X^{6}-1\right) /(X-1)=(X+1)(X-3)(X-4)(X-9)(X-10),
$$

it follows that the eigenspaces $V_{j}^{(\lambda)}$ corresponding to the eigenvalues $\lambda=-1,3,4,9$, 10 are 1-dimensional. In particular, $\operatorname{dim} V_{j}^{(4)}=1$.

Lemma 7.3. Under the above notation we have

$$
V_{j}^{(4)} \cap\left(U_{E_{j}}^{\prime} / U_{E_{j}}^{13}\right) \subseteq \varepsilon_{2}\left(U_{E_{j}}^{\prime} / U_{E_{j}}^{13}\right),
$$

and if in $F_{j}$ there are no nontrivial singular primary units, the equality holds in (7.3).

Proof. From Lemma 7.1 (ii) it follows that if $u \in V_{j}^{(4)}$, i.e., $\sigma(u)=u^{4}$, then $\varepsilon_{2} u=u$. Hence $u \in \varepsilon_{2}\left(U_{E_{j}}^{\prime} / U_{E_{j}}^{13}\right)$.

Conversely, if $u \in U_{E_{j}}^{\prime} / U_{E_{j}}^{13}, \varepsilon_{2} u=u$, and there are no nontrivial singular primary units in $F_{j}$, then the assumption (7.1) of Lemma 7.1 is satisfied, and by part (ii) of that lemma we get $\sigma(u)=u^{4}$, i.e., $u \in V_{j}^{(4)}$.

It turns out that the 13-primary parts of the class groups of the fields $F_{j}$ and $E_{j}, j=1,2$, are trivial for all fields $F$ mentioned above. Moreover, in $F_{j}, j=1,2$, there are no nontrivial singular primary units, with one exception: $p=487$, and the field $E_{2}$. Nevertheless, in this exceptional case, we have $N_{E_{2} / F_{2}}(u)=1$ for every $u \in U_{E_{2}}^{\prime} / U_{E_{2}}^{13}$. Therefore, the equality always holds in (7.3), and this makes it possible to determine the 13-rank of $K_{2} \mathcal{O}_{F}$ for every field in Table 1.

First for every field $E_{j}$ we have determined a unit $u$ generating the 1-dimensional subspace $V_{j}^{(4)}$, and next we have verified whether $u$ is singular primary.

Similarly to the case $q=7$ the minimal polynomial for $\rho_{j}$ is an Eisenstein polynomial with respect to $q=13$, and every unit $u \in U_{E_{j}}$ can be written in the form

$$
u=a_{0}+a_{1} x+\cdots+a_{5} x^{5},
$$

where $x=\rho_{j}, a_{0}, \ldots, a_{5} \in \mathbb{Q}$, and $v_{13}\left(a_{0}\right)=0$. We can assume that $a_{0} \equiv 1$ (mod 13$)$, replacing $u$ by $u^{12}$. 
Then $u$ is a singular primary unit iff $a_{0} \equiv 1\left(\bmod 13^{2}\right)$ and $a_{i} \equiv 0(\bmod 13)$ for $i=1, \ldots, 5$. Table 3 (at the end of the paper) gives the results concerning the 13-rank of $K_{2} \mathcal{O}_{F}$. We use the following notation: $v_{13}:=v_{13}\left(\# K_{2} \mathcal{O}_{F}\right), d_{j}:=$ $\operatorname{dim}\left(V_{j}^{(4)} \cap\left(U_{E_{j}}^{\prime} / U_{E_{j}}^{13}\right)\right)$, and $\left(K_{2} \mathcal{O}_{F}\right)_{13}$ denotes the 13-primary part of $K_{2} \mathcal{O}_{F}$.

\section{Description of Table 1}

The first column of the table lists all primes $p \equiv 1(\bmod 6), p<5000$, and the second the corresponding values of $A$. The third and fourth columns give the orders of $K_{2} \mathcal{O}_{F}$; moreover, the fourth column provides information about the structure of the group $K_{2} \mathcal{O}_{F}$. To save space we use the following convention. If the order of a group is written in the form $\left(n_{1}\right)^{k_{1}}\left(n_{2}\right)^{k_{2}} \ldots$, where $\left(n_{j}\right)^{k_{j}}$ is in roman font, it means that the group is isomorphic to the product of $k_{1}$ copies of $\mathbb{Z} / n_{1}, k_{2}$ copies of $\mathbb{Z} / n_{2}$, etc. However, if a factor $\left(n_{j}^{k_{j}}\right)$ is written in bold type, it means that there is a direct summand of order $n_{j}^{k_{j}}$, but its structure is unknown. Thus $(2)^{2}$ means $\mathbb{Z} / 2 \times \mathbb{Z} / 2$, and $\left(2^{2}\right)$ means $\mathbb{Z} / 4$, but $\left(\mathbf{3 1}^{\mathbf{2}}\right)$ means a group of order $31^{2}$, i.e., one of the groups $\mathbb{Z} / 31 \times \mathbb{Z} / 31$ and $\mathbb{Z} / 31^{2}$.

The fifth column of the table gives the class group of $F$, provided it is nontrivial. We use the same convention here, e.g. $(2)^{2}$ means $\mathbb{Z} / 2 \times \mathbb{Z} / 2$.

The structure of the 2-primary part of $K_{2} \mathcal{O}_{F}$ can be determined as follows. The ideal (2) splits in $\mathcal{O}_{F}$ into nonprincipal ideals iff $p$ belongs to the following list: $277,397,1399,1459,1699,1777,1789,1831,2689,3271,4339,4567$. It turns out that in all these cases the 2-primary part of the group $\operatorname{Cl}\left(\mathcal{O}_{F, 2}\right)$ is trivial, even for $p=1777$, when $C l\left(\mathcal{O}_{F}\right)=\mathbb{Z} / 4 \times \mathbb{Z} / 4$.

For other fields in the table, 2-rank $C l\left(\mathcal{O}_{F, 2}\right)=2$-rank $C l\left(\mathcal{O}_{F}\right)$. Consequently, by Lemma 3.2, 2-rank $\left(K_{2} \mathcal{O}_{F}\right)=3$ or 5 for every $p<5000$.

Now we can apply the second inequality of Corollary 3.6. Namely, it turns out that the fundamental unit $\varepsilon_{1}$ is totally positive iff $p=1009$ or 4567 (for $p<$ $5000)$. Fortunately, for these two fields the corresponding elements $\gamma_{j}$ are not totally positive. Then by Corollary 3.6, 4-rank $\left(K_{2} \mathcal{O}_{F}\right) \leq 2-\operatorname{rank}\left(K_{2} \mathcal{O}_{F}\right)-3$. Hence $4-\operatorname{rank}\left(K_{2} \mathcal{O}_{F}\right)=0$ or 2, and if 2-rank $\left(K_{2} \mathcal{O}_{F}\right)=3$, then 4-rank $\left(K_{2} \mathcal{O}_{F}\right)=0$, for $p<5000$. It follows that if $e_{2}:=2$-rank $\left(K_{2} \mathcal{O}_{F}\right)$ and $v_{2}\left(\# K_{2} \mathcal{O}_{F}\right)=r$, then the 2-primary part of $K_{2} \mathcal{O}_{F}$ is isomorphic to

$$
(\mathbb{Z} / 2)^{e_{2}-2} \times\left(\mathbb{Z} / 2^{s}\right)^{2}, \quad \text { where } s=\left(r-e_{2}\right) / 2+1 .
$$

To determine the structure of the 3-primary part of $K_{2} \mathcal{O}_{F}$ we use Theorem 4.7: 3 -rank $\left(K_{2} \mathcal{O}_{F}\right)=3$-rank $\left(C l\left(\mathcal{O}_{E}\right)\right)$. It can be verified that for all $p<5000$ we have 3 -rank $\left(C l\left(\mathcal{O}_{E}\right)\right) \leq 2$. Then the structure of $K_{2} \mathcal{O}_{F}$ is determined uniquely, unless 3 -rank $\left(C l\left(\mathcal{O}_{E}\right)\right)=2$ and $v_{3}\left(\# K_{2} \mathcal{O}_{F}\right)>3$. The latter is the case for $p=1747$, $2593,3061,4789$, where the 9-rank of $K_{2} \mathcal{O}_{F}$ is unknown.

The following facts have been verified for $p<5000$, but we do not know if they are true in general.

(i) If $9 \mid \# K_{2} \mathcal{O}_{F}$, then $3-\operatorname{rank}\left(K_{2} \mathcal{O}_{F}\right)=2$.

(ii) $3 \| \# K_{2} \mathcal{O}_{F}$ iff $3 \| \# \mathrm{Cl}\left(\mathcal{O}_{E}\right)$.

(iii) $9 \| \# K_{2} \mathcal{O}_{F}$ iff $\operatorname{Syl}_{3} C l\left(\mathcal{O}_{E}\right)=\mathbb{Z} / 3 \times \mathbb{Z} / 3$.

(iv) $27 \mid \# K_{2} \mathcal{O}_{F}$ iff $\mathbb{Z} / 3 \times \mathbb{Z} / 9 \subseteq C l\left(\mathcal{O}_{E}\right)$.

Thus the 3-primary part of the group $\mathrm{Cl}\left(\mathcal{O}_{E}\right)$ is never cyclic provided $9 \mid \# K_{2} \mathcal{O}_{F}$. 
TABLE 1.

\begin{tabular}{|c|c|c|c|c|}
\hline$p$ & $A$ & $\# K_{2} \mathcal{O}_{F}$ & $K_{2} \mathcal{O}_{F}$ & $C l\left(\mathcal{O}_{F}\right)$ \\
\hline$\overline{\overline{7}}$ & 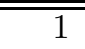 & 8 & $\overline{(2)^{3}}$ & \\
\hline 13 & -5 & 8 & $(2)^{3}$ & \\
\hline 19 & 7 & 24 & $(2)^{3}(3)$ & \\
\hline 31 & 4 & 224 & $(2)^{5}(7)$ & \\
\hline 37 & -11 & 168 & $(2)^{3}(3)(7)$ & \\
\hline 43 & -8 & 608 & $(2)^{5}(19)$ & \\
\hline 61 & 1 & 1064 & $(2)^{3}(7)(19)$ & \\
\hline 67 & -5 & 1544 & $(2)^{3}(193)$ & \\
\hline 73 & 7 & 1896 & $(2)^{3}(3)(79)$ & \\
\hline 79 & -17 & 1592 & $(2)^{3}(199)$ & \\
\hline 97 & 19 & 2936 & $(2)^{3}(367)$ & \\
\hline 103 & 13 & 5096 & $(2)^{3}\left(7^{2}\right)(13)$ & \\
\hline 109 & -2 & 9888 & $(2)^{5}(3)(103)$ & \\
\hline 127 & -20 & 17376 & $(2)^{5}(3)(181)$ & \\
\hline 139 & -23 & 8600 & $(2)^{3}(5)^{2}(43)$ & \\
\hline 151 & 19 & 15848 & $(2)^{3}(7)(283)$ & \\
\hline 157 & -14 & 30944 & $(2)^{5}(967)$ & \\
\hline 163 & 25 & 16224 & $(2)^{5}(3)\left(13^{2}\right)$ & $(2)^{2}$ \\
\hline 181 & 7 & 23064 & $(2)^{3}(3)\left(\mathbf{3 1}^{\mathbf{2}}\right)$ & \\
\hline 193 & -23 & 34328 & $(2)^{3}(7)(613)$ & \\
\hline 199 & -11 & 29448 & $(2)^{3}(3)^{2}(409)$ & \\
\hline 211 & 13 & 35528 & $(2)^{3}(4441)$ & \\
\hline 223 & 28 & 90272 & $(2)^{5}(7)(13)(31)$ & \\
\hline 229 & 22 & 94304 & $(2)^{5}(7)(421)$ & \\
\hline 241 & -17 & 51464 & $(2)^{3}(7)(919)$ & \\
\hline 271 & -29 & 93816 & $(2)^{3}(3)^{2}(1303)$ & \\
\hline 277 & -26 & 162944 & $(2)^{3}\left(2^{2}\right)^{2}(19)(67)$ & $(2)^{2}$ \\
\hline 283 & -32 & 171104 & $(2)^{5}(5347)$ & \\
\hline 307 & 16 & 311424 & $(2)^{3}\left(2^{2}\right)^{2}(3)(811)$ & \\
\hline 313 & -35 & 119336 & $(2)^{3}(7)(2131)$ & (7) \\
\hline 331 & 1 & 122888 & $(2)^{3}(15361)$ & \\
\hline 337 & -5 & 152264 & $(2)^{3}(7)(2719)$ & \\
\hline 349 & 37 & 141728 & $(2)^{5}(43)(103)$ & $(2)^{2}$ \\
\hline 367 & -35 & 270392 & $(2)^{3}(73)(463)$ & \\
\hline 373 & 13 & 184232 & $(2)^{3}(23029)$ & \\
\hline 379 & -29 & 198744 & $(2)^{3}(3)\left(7^{2}\right)(13)^{2}$ & \\
\hline 397 & 34 & 472704 & $(2)^{3}\left(2^{2}\right)^{2}(3)(1231)$ & $(2)^{2}$ \\
\hline 409 & 31 & 260648 & $(2)^{3}(31)(1051)$ & \\
\hline 421 & 19 & 261704 & $(2)^{3}(32713)$ & \\
\hline 433 & -2 & 606336 & $(2)^{3}\left(2^{2}\right)^{2}(3)(1579)$ & \\
\hline 439 & 28 & 952448 & $(2)^{3}\left(2^{2}\right)^{2}(7)(1063)$ & \\
\hline 457 & 10 & 832544 & $(2)^{5}(26017)$ & \\
\hline
\end{tabular}


TABLE 1. (continued)

\begin{tabular}{|c|c|c|c|c|}
\hline$p$ & $A$ & $\# K_{2} \mathcal{O}_{F}$ & $K_{2} \mathcal{O}_{F}$ & $\mathrm{Cl}\left(\mathcal{O}_{F}\right)$ \\
\hline 463 & -23 & 338600 & 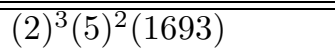 & \\
\hline 487 & 25 & 450216 & $(2)^{3}(3)^{2}(13)^{2}(37)$ & \\
\hline 499 & -32 & 1344032 & $\left(2^{5}\right)(97)(433)$ & \\
\hline 523 & 43 & 674856 & $(2)^{3}(3)^{2}(7)(13)(103)$ & \\
\hline 541 & -29 & 543528 & $(2)^{3}(3)^{2}(7549)$ & \\
\hline 547 & 1 & 746144 & $(2)^{5}(7)(3331)$ & $(2)^{2}$ \\
\hline 571 & 31 & 635768 & $(2)^{3}(7)(11353)$ & \\
\hline 577 & -11 & 908088 & $(2)^{3}(3)(157)(241)$ & \\
\hline 601 & -26 & 1649504 & $(2)^{5}(19)(2713)$ & \\
\hline 607 & 49 & 759776 & $(2)^{5}(23743)$ & $(2)^{2}$ \\
\hline 613 & -47 & 1067928 & $(2)^{3}(3)(44497)$ & \\
\hline 619 & -17 & 1115912 & $(2)^{3}(7)(19927)$ & \\
\hline 631 & 43 & 905064 & $(2)^{3}(3)(43)(877)$ & \\
\hline 643 & 40 & 3180512 & $(2)^{5}(99391)$ & \\
\hline 661 & 49 & 1434104 & $(2)^{3}(7)(25609)$ & \\
\hline 673 & 37 & 1043672 & $(2)^{3}(7)(18637)$ & \\
\hline 691 & -8 & 2807168 & $(2)^{3}\left(2^{2}\right)^{2}(7)(13)(241)$ & \\
\hline 709 & -53 & 1159328 & $(2)^{5}(36229)$ & $(2)^{2}$ \\
\hline 727 & -44 & 4195808 & $(2)^{5}(19)(67)(103)$ & \\
\hline 733 & -50 & 3434144 & $(2)^{5}(7)(15331)$ & \\
\hline 739 & 16 & 3466752 & $(2)^{3}\left(2^{3}\right)^{2}(3)(37)(61)$ & \\
\hline 751 & -41 & 1439672 & $(2)^{3}(13)(109)(127)$ & \\
\hline 757 & -29 & 1975272 & $(2)^{3}(3)\left(13^{2}\right)(487)$ & \\
\hline 769 & 49 & 1763528 & $(2)^{3}(13)(31)(547)$ & \\
\hline 787 & 31 & 2218136 & $(2)^{3}(19)(14593)$ & \\
\hline 811 & -56 & 4291872 & $(2)^{5}(3)(13)(19)(181)$ & \\
\hline 823 & -5 & 2084600 & $(2)^{3}(5)^{2}(7)(1489)$ & \\
\hline 829 & 7 & 1997304 & $(2)^{3}(3)(83221)$ & \\
\hline 853 & -35 & 3450272 & $(2)^{5}(7)(73)(211)$ & $(2)^{2}$ \\
\hline 859 & 13 & 2109992 & $(2)^{3}(73)(3613)$ & \\
\hline 877 & -59 & 2241848 & $(2)^{3}(7)\left(7^{2}\right)(19)(43)$ & (7) \\
\hline 883 & -47 & 2377608 & $(2)^{3}(3)(157)(631)$ & \\
\hline 907 & 19 & 2474312 & $(2)^{3}(309289)$ & \\
\hline 919 & 52 & 8472672 & $(2)^{5}(3)^{2}(13)(31)(73)$ & \\
\hline 937 & 61 & 2641632 & $(2)^{5}(3)(7)(3931)$ & $(2)^{2}$ \\
\hline 967 & -41 & 4288664 & $(2)^{3}(31)(17293)$ & \\
\hline 991 & 61 & 4490088 & $(2)^{3}(3)(61)(3067)$ & \\
\hline 997 & 10 & 11895008 & $(2)^{5}(371719)$ & \\
\hline 1009 & 43 & 4821504 & $(2)^{3}\left(2^{3}\right)^{2}(3)(43)(73)$ & $(2)^{2}$ \\
\hline 1021 & -14 & 12182144 & $(2)^{3}\left(2^{2}\right)^{2}(13)(7321)$ & \\
\hline 1033 & -53 & 3748376 & $(2)^{3}(103)(4549)$ & \\
\hline 1039 & -59 & 4077704 & $(2)^{3}(19)(139)(193)$ & \\
\hline
\end{tabular}


TABle 1. (continued)

\begin{tabular}{|c|c|c|c|c|}
\hline$p$ & $A$ & $\# K_{2} \mathcal{O}_{F}$ & $K_{2} \mathcal{O}_{F}$ & $C l\left(\mathcal{O}_{F}\right)$ \\
\hline$\overline{\overline{1051}}$ & 64 & 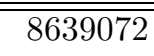 & 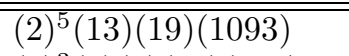 & \\
\hline 1063 & -65 & 4472664 & $(2)^{3}(3)(7)(79)(337)$ & (13) \\
\hline 1069 & -62 & 9159584 & $(2)^{5}(7)(103)(397)$ & \\
\hline 1087 & 55 & 5176088 & $(2)^{3}(647011)$ & \\
\hline 1093 & 22 & 14307488 & $(2)^{5}(13)(163)(211)$ & \\
\hline 1117 & -65 & 7521864 & $(2)^{3}(3)(7)(44773)$ & \\
\hline 1123 & -35 & 5642744 & $(2)^{3}(31)(61)(373)$ & \\
\hline 1129 & 67 & 4681832 & $(2)^{3}(37)(15817)$ & (7) \\
\hline 1153 & 7 & 5324856 & $(2)^{3}(3)(127)(1747)$ & \\
\hline 1171 & -11 & 5391816 & $(2)^{3}(3)(271)(829)$ & \\
\hline 1201 & -59 & 6078488 & $(2)^{3}(13)(211)(277)$ & \\
\hline 1213 & -17 & 5861816 & $(2)^{3}(853)(859)$ & \\
\hline 1231 & 19 & 6344408 & $(2)^{3}(7)(277)(409)$ & \\
\hline 1237 & -41 & 6324152 & $(2)^{3}(790519)$ & \\
\hline 1249 & -53 & 8869736 & $(2)^{3}(1108717)$ & \\
\hline 1279 & 43 & 6901272 & $(2)^{3}(3)^{2}(7)(13693)$ & \\
\hline 1291 & 67 & 7776776 & $(2)^{3}(7)(19)(7309)$ & \\
\hline 1297 & 25 & 8220744 & $(2)^{3}(3)\left(3^{2}\right)(7)(5437)$ & \\
\hline 1303 & 55 & 11756264 & $(2)^{3}(13)(113041)$ & \\
\hline 1321 & -71 & 10453448 & $(2)^{3}(31)(61)(691)$ & \\
\hline 1327 & 4 & 18672032 & $(2)^{5}(583501)$ & \\
\hline 1381 & 31 & 8621288 & $(2)^{3}(13)(19)(4363)$ & \\
\hline 1399 & -68 & 30029696 & $(2)^{3}\left(2^{2}\right)^{2}(283)(829)$ & $(2)^{2}$ \\
\hline 1423 & -20 & 26058336 & $(2)^{5}(3)(521)^{2}$ & \\
\hline 1429 & -71 & 10536248 & $(2)^{3}(1317031)$ & \\
\hline 1447 & -35 & 12013736 & $(2)^{3}(7)(214531)$ & \\
\hline 1453 & 67 & 10408088 & $(2)^{3}(1301011)$ & \\
\hline 1459 & -56 & 28097664 & $(2)^{3}\left(2^{2}\right)^{2}(3)(7)(10453)$ & (13) \\
\hline 1471 & 76 & 24495200 & $(2)^{5}(5)^{2}(67)(457)$ & \\
\hline 1483 & 37 & 10679384 & $(2)^{3}(37)(109)(331)$ & \\
\hline 1489 & -77 & 11908904 & $(2)^{3}(7)(109)(1951)$ & (19) \\
\hline 1531 & 7 & 19844424 & $(2)^{3}(3)^{2}(367)(751)$ & \\
\hline 1543 & -77 & 18481832 & $(2)^{3}(19)(121591)$ & \\
\hline 1549 & -11 & 20078664 & $(2)^{3}(3)(836611)$ & \\
\hline 1567 & 79 & 12582024 & $(2)^{3}(3)(7)^{2}(13)(823)$ & (7) \\
\hline 1579 & -32 & 32194208 & $(2)^{5}(19)(52951)$ & \\
\hline 1597 & -50 & 48989024 & $(2)^{5}\left(7^{2}\right)(157)(199)$ & \\
\hline 1609 & 19 & 21766568 & $(2)^{3}(2720821)$ & \\
\hline 1621 & 79 & 19523448 & $(2)^{3}(3)^{2}(7)(38737)$ & \\
\hline 1627 & -80 & 38016992 & $(2)^{5}(13)(91387)$ & \\
\hline 1657 & 70 & 41556192 & $(2)^{5}(3)(19)(22783)$ & \\
\hline 1663 & 73 & 15879128 & $(2)^{3}(1984891)$ & \\
\hline
\end{tabular}


TABle 1. (continued)

\begin{tabular}{|r|r|r|l|c|}
\hline$p$ & $A$ & $\# K_{2} \mathcal{O}_{F}$ & $K_{2} \mathcal{O}_{F}$ & $C l\left(\mathcal{O}_{F}\right)$ \\
\hline \hline 1669 & 67 & 21204728 & $(2)^{3}(139)(19069)$ & \\
1693 & -47 & 16202232 & $(2)^{3}(3)^{2}(307)(733)$ & \\
1699 & 64 & 42115712 & $(2)^{3}\left(2^{2}\right)^{2}(151)(2179)$ & $(2)^{2}$ \\
1723 & 40 & 46730528 & $(2)^{5}(13)^{2}(8641)$ & \\
1741 & 49 & 18534152 & $(2)^{3}\left(7^{2}\right)(13)(3637)$ & \\
1747 & 61 & 17597736 & $(2)^{3}\left(3^{4}\right)(13)(2089)$ & \\
1753 & 10 & 46850528 & $(2)^{5}(1464079)$ & \\
1759 & 31 & 28347032 & $(2)^{3}(7)(37)(13681)$ & \\
1777 & -14 & 45192704 & $(2)^{3}\left(2^{3}\right)^{2}(61)(1447)$ & $\left(2^{2}\right)^{2}$ \\
1783 & -83 & 26028744 & $(2)^{3}(3)(7)(154933)$ & \\
1789 & 82 & 44748416 & $(2)^{3}\left(2^{2}\right)^{2}(73)(4789)$ & $(2)^{2}$ \\
1801 & -74 & 44090592 & $(2)^{5}(3)(7)\left(7^{2}\right)(13)(103)$ & \\
1831 & -68 & 53752736 & $(2)^{5}(1679773)$ & $(7)$ \\
1861 & 37 & 33785624 & $(2)^{3}(853)(4951)$ & \\
1867 & 85 & 34006856 & $(2)^{3}\left(13^{2}\right)(25153)$ & \\
1873 & -65 & 25249128 & $(2)^{3}(3)(31)(33937)$ & $(2)^{2}$ \\
1879 & 73 & 30150368 & $(2)^{5}(942199)$ & \\
1933 & -62 & 79342496 & $(2)^{5}(2479453)$ & $(2)^{2}$ \\
1951 & 1 & 24210656 & $(2)^{5}(61)(79)(157)$ & $(2)^{2}$ \\
1987 & -89 & 25099304 & $(2)^{3}(19)(61)(2707)$ & \\
1993 & 13 & 26971448 & $(2)^{3}(7)(481633)$ & \\
1999 & 52 & 64523808 & $(2)^{5}(3)^{2}(224041)$ & \\
2011 & -59 & 26608088 & $(2)^{3}(13)(255847)$ & \\
2017 & 34 & 62057088 & $(2)^{3}\left(2^{2}\right)^{2}(3)^{2}(103)(523)$ & \\
2029 & -77 & 41567288 & $(2)^{3}\left(7^{2}\right)(19)(5581)$ & \\
2053 & -83 & 30386616 & $(2)^{3}(3)(13)(17)^{2}(337)$ & \\
2083 & -23 & 29384504 & $(2)^{3}(733)(5011)$ & \\
2089 & -38 & 69126432 & $(2)^{5}(3)(223)(3229)$ & \\
2113 & 82 & 70391072 & $(2)^{5}(61)(36061)$ & \\
2131 & 91 & 48170528 & $(2)^{5}(7)^{2}(31)(991)$ & \\
2137 & 85 & 38567048 & $(2)^{3}(13)(370837)$ & \\
2143 & -92 & 73613664 & $(2)^{5}(3)\left(3^{2}\right)(85201)$ & \\
2161 & -29 & 32856264 & $(2)^{3}(3)^{2}\left(7^{2}\right)(67)(139)$ & \\
2179 & 88 & 113959392 & $(2)^{5}(3)(157)(7561)$ & \\
2203 & -8 & 116452064 & $(2)^{5}(19)(191533)$ & \\
2221 & -53 & 56434376 & $(2)^{3}(7054297)$ & \\
2239 & 91 & 44756552 & $(2)^{3}(19)(277)(1063)$ & \\
2251 & 16 & 125175264 & $(2)^{5}(3)(127)(10267)$ & \\
2269 & -83 & 53021592 & $(2)^{3}(3)^{2}(13)(37)(1531)$ & \\
2281 & -86 & 90707552 & $(2)^{5}(13)(218047)$ & \\
\end{tabular}


TABle 1. (continued)

\begin{tabular}{|c|c|c|c|c|}
\hline$p$ & $A$ & $\# K_{2} \mathcal{O}_{F}$ & $K_{2} \mathcal{O}_{F}$ & $\mathrm{Cl}\left(\mathcal{O}_{F}\right)$ \\
\hline 2287 & -20 & 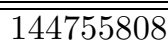 & 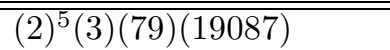 & \\
\hline 2293 & 37 & 39299096 & $(2)^{3}(991)(4957)$ & \\
\hline 2311 & -89 & 42889952 & $(2)^{5}(7)(191473)$ & $(2)^{2}$ \\
\hline 2341 & -74 & 139225632 & $(2)^{5}(3)(7)(13)(15937)$ & \\
\hline 2347 & 64 & 105761888 & $(2)^{5}(1039)(3181)$ & \\
\hline 2371 & -41 & 43322888 & $(2)^{3}(7)\left(\mathbf{1 9}^{\mathbf{2}}\right)(2143)$ & \\
\hline 2377 & 79 & 43970328 & $(2)^{3}(3)^{2}(610699)$ & \\
\hline 2383 & 28 & 157511072 & $(2)^{5}(37)(133033)$ & \\
\hline 2389 & -59 & 70897688 & $(2)^{3}(97)(211)(433)$ & \\
\hline 2437 & 1 & 48333848 & $(2)^{3}(6041731)$ & (7) \\
\hline 2467 & -11 & 50148936 & $(2)^{3}(3)\left(3^{2}\right)(232171)$ & \\
\hline 2473 & 73 & 50147624 & $(2)^{3}(67)(93559)$ & \\
\hline 2503 & -47 & 50764200 & $(2)^{3}(3)(5)^{2}(19)(61)(73)$ & \\
\hline 2521 & 97 & 59782056 & $(2)^{3}(3)(19)(131101)$ & \\
\hline 2539 & -83 & 53568600 & $(2)^{3}(3)(5)^{2}(19)(37)(127)$ & \\
\hline 2551 & 49 & 57461816 & $(2)^{3}(7182727)$ & \\
\hline 2557 & -101 & 53301624 & $(2)^{3}(3)(439)(5059)$ & (7) \\
\hline 2593 & 25 & 63726264 & $(2)^{3}\left(\mathbf{3}^{\mathbf{6}}\right)\left(7^{2}\right)(223)$ & \\
\hline 2617 & 91 & 90367832 & $(2)^{3}(11295979)$ & \\
\hline 2647 & -29 & 60219096 & $(2)^{3}(3)(7)(358447)$ & \\
\hline 2659 & 103 & 63408968 & $(2)^{3}(7)(73)(15511)$ & (19) \\
\hline 2671 & -44 & 210615392 & $(2)^{5}(13)(283)(1789)$ & \\
\hline 2677 & 31 & 62355176 & $(2)^{3}(13)(61)(9829)$ & \\
\hline 2683 & 97 & 68405928 & $(2)^{3}(3)(19)(67)(2239)$ & \\
\hline 2689 & -62 & 148030976 & $(2)^{3}\left(2^{3}\right)^{2}(19)(15217)$ & $(2)^{2}$ \\
\hline 2707 & 55 & 74568200 & $(2)^{3}(5)^{2}\left(7^{3}\right)(1087)$ & \\
\hline 2713 & 103 & 91568648 & $(2)^{3}(103)(111127)$ & \\
\hline 2719 & -101 & 74672952 & $(2)^{3}(3)(127)(24499)$ & \\
\hline 2731 & -104 & 154184576 & $(2)^{3}\left(2^{2}\right)^{2}\left(7^{2}\right)(13)(31)(61)$ & \\
\hline 2749 & -14 & 191244800 & $(2)^{3}\left(2^{3}\right)^{2}(5)^{2}(67)(223)$ & \\
\hline 2767 & 76 & 176114144 & $(2)^{5}(5503567)$ & \\
\hline 2791 & -92 & 188965248 & $(2)^{3}\left(2^{2}\right)^{2}(3)(163)(3019)$ & \\
\hline 2797 & -89 & 73500128 & $(2)^{5}\left(13^{2}\right)(13591)$ & $(2)^{2}$ \\
\hline 2803 & -95 & 117740000 & $(2)^{5}\left(\mathbf{5}^{\mathbf{4}}\right)(7)(29)^{2}$ & $(2)^{2}$ \\
\hline 2833 & -98 & 187258496 & $(2)^{3}\left(2^{2}\right)^{2}(1462957)$ & \\
\hline 2851 & 73 & 119950136 & $(2)^{3}(673)(22279)$ & \\
\hline 2857 & -41 & 75490952 & $(2)^{3}(19)(31)(37)(433)$ & \\
\hline 2887 & 91 & 86493368 & $(2)^{3}(13)(349)(2383)$ & \\
\hline 2917 & 106 & 186688224 & $(2)^{5}(3)^{2}(19)(109)(313)$ & \\
\hline 2953 & 70 & 231006048 & $(2)^{5}(3)(7)(13)(31)(853)$ & \\
\hline 2971 & -56 & 298295808 & $(2)^{3}\left(2^{3}\right)^{2}(3)(194203)$ & \\
\hline
\end{tabular}


TABle 1. (continued)

\begin{tabular}{|c|c|c|c|c|}
\hline$p$ & $A$ & $\# K_{2} \mathcal{O}_{F}$ & $K_{2} \mathcal{O}_{F}$ & $C l\left(\mathcal{O}_{F}\right)$ \\
\hline 3001 & -77 & 151503416 & 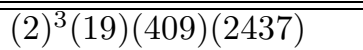 & \\
\hline 3019 & 13 & 136579688 & $(2)^{3}(7)(907)(2689)$ & \\
\hline 3037 & 49 & 96292448 & $(2)^{5}\left(7^{3}\right)(31)(283)$ & $(2)^{2}$ \\
\hline 3049 & -17 & 139208552 & $(2)^{3}(7)(2485867)$ & \\
\hline 3061 & -38 & 245335392 & $(2)^{5}\left(\mathbf{3}^{\mathbf{4}}\right)(94651)$ & \\
\hline 3067 & 19 & 143062472 & $(2)^{3}(7)(367)(6961)$ & \\
\hline 3079 & 79 & 152143368 & $(2)^{3}(3)(13)(337)(1447)$ & \\
\hline 3109 & -23 & 146303288 & $(2)^{3}(109)(167779)$ & \\
\hline 3121 & -89 & 100077224 & $(2)^{3}(13)(379)(2539)$ & \\
\hline 3163 & 112 & 252655616 & $(2)^{3}\left(2^{4}\right)^{2}(19)(43)(151)$ & \\
\hline 3169 & 97 & 105161208 & $(2)^{3}(3)(907)(4831)$ & \\
\hline 3181 & 94 & 341303936 & $(2)^{3}\left(2^{2}\right)^{2}(139)(19183)$ & \\
\hline 3187 & -29 & 162777192 & $(2)^{3}(3)(619)(10957)$ & \\
\hline 3217 & 31 & 164205608 & $(2)^{3}(7)(79)(37117)$ & (7) \\
\hline 3229 & 46 & 291821984 & $(2)^{5}(67)(136111)$ & \\
\hline 3253 & -113 & 156813992 & $(2)^{3}(19)(37)(27883)$ & \\
\hline 3259 & 88 & 285120384 & $(2)^{3}\left(2^{2}\right)^{2}(3)(19)(39079)$ & \\
\hline 3271 & 4 & 272604032 & $(2)^{3}\left(2^{2}\right)^{2}(2129719)$ & $(2)^{2}$ \\
\hline 3301 & 109 & 122600504 & $(2)^{3}(13)(1178851)$ & \\
\hline 3307 & -59 & 118606136 & $(2)^{3}(11)^{2}(122527)$ & \\
\hline 3313 & 115 & 132209448 & $(2)^{3}(3)\left(7^{2}\right)(19)(61)(97)$ & (19) \\
\hline 3319 & 37 & 183639176 & $(2)^{3}(7)(23)^{2}(6199)$ & \\
\hline 3331 & 16 & 282038784 & $(2)^{3}\left(2^{3}\right)^{2}(3)(139)(1321)$ & \\
\hline 3343 & -68 & 400784864 & $(2)^{5}(31)(404017)$ & \\
\hline 3361 & -113 & 138729752 & $(2)^{3}(7)(2477317)$ & \\
\hline 3373 & -98 & 436401824 & $(2)^{5}(379)(35983)$ & \\
\hline 3391 & -116 & 293541152 & $(2)^{5}(9173161)$ & \\
\hline 3433 & -77 & 143234072 & $(2)^{3}(1483)(12073)$ & \\
\hline 3457 & -110 & 362770944 & $(2)^{3}\left(2^{3}\right)^{2}(3)(103)(2293)$ & \\
\hline 3463 & 28 & 344540192 & $(2)^{5}(10766881)$ & \\
\hline 3469 & 103 & 138196856 & $(2)^{3}(7)^{2}(352543)$ & \\
\hline 3499 & -89 & 223275464 & $(2)^{3}(37)(73)(10333)$ & \\
\hline 3511 & 79 & 143220168 & $(2)^{3}\left(3^{)} 2(7)(13)(21859)\right.$ & \\
\hline 3517 & 109 & 201534944 & $(2)^{5}(13)(484459)$ & $(2)^{2}$ \\
\hline 3529 & -47 & 217436856 & $(2)^{3}(3)(7)(13)(99559$ & (19) \\
\hline 3541 & 58 & 395526368 & $(2)^{5}(12360199)$ & \\
\hline 3547 & -119 & 153726792 & $(2)^{3}(3)(6405283)$ & (19) \\
\hline 3559 & 67 & 152045816 & $(2)^{3}(13)(1461979)$ & \\
\hline 3571 & 1 & 147892568 & $(2)^{3}(31)(596341)$ & (7) \\
\hline 3583 & 7 & 161986392 & $(2)^{3}\left(\mathbf{3}^{\mathbf{4}}\right)(457)(547)$ & \\
\hline 3607 & -116 & 504481952 & $(2)^{5}(13)(1212697)$ & \\
\hline
\end{tabular}


TABle 1. (continued)

\begin{tabular}{|r|r|r|l|l|}
\hline$p$ & $A$ & $\# K_{2} \mathcal{O}_{F}$ & $K_{2} \mathcal{O}_{F}$ & $C l\left(\mathcal{O}_{F}\right)$ \\
\hline \hline 3613 & 13 & 154051112 & $(2)^{3}(43)(447823)$ & \\
3631 & 76 & 518241248 & $(2)^{5}(7)^{2}(67)(4933)$ & \\
3637 & 115 & 192358872 & $(2)^{3}(3)^{2}(2671651)$ & \\
3643 & -17 & 158957528 & $(2)^{3}(19869691)$ & \\
3673 & -83 & 159534648 & $(2)^{3}(3)^{2}(7)\left(13^{2}\right)(1873)$ & \\
3691 & -101 & 163217832 & $(2)^{3}(3)(6800743)$ & \\
3697 & -71 & 167071592 & $(2)^{3}(409)(51061)$ & \\
3709 & -119 & 203851464 & $(2)^{3}(3)(8493811)$ & \\
3727 & 25 & 188158752 & $(2)^{5}(3)^{2}(103)(6343)$ & $(2)^{2}$ \\
3733 & 55 & 290980136 & $(2)^{3}(19)(31)(37)(1669)$ & \\
3739 & -113 & 247660184 & $(2)^{3}(30957523)$ & \\
3769 & 73 & 172890536 & $(2)^{3}(7)(13)(237487)$ & \\
3793 & 103 & 178884776 & $(2)^{3}(7)(3194371)$ & \\
3823 & 100 & 513848672 & $(2)^{5}(109)(147319)$ & \\
3847 & -59 & 283234088 & $(2)^{3}(1621)(21841)$ & \\
3853 & 115 & 299061672 & $(2)^{3}(3)(7)(13)(19)(7207)$ & \\
3877 & -35 & 228407816 & $\left.(2)^{3}(7)(7)^{2}\right)(13)(19)(337)$ & \\
3889 & -2 & 639625056 & $(2)^{5}(3)(7)(103)(9241)$ & \\
3907 & 61 & 288686664 & $(2)^{3}(3)^{2}(7)(572791)$ & \\
3919 & -77 & 213262376 & $(2)^{3}(37)(720481)$ & \\
3931 & -89 & 198399272 & $(2)^{3}(24799909)$ & \\
3943 & 52 & 477244032 & $(2)^{3}\left(2^{2}\right)^{2}(3)(1242823)$ & \\
3967 & -125 & 325012856 & $(2)^{3}(7)(97)(59833)$ & \\
4003 & -107 & 215594744 & $(2)^{3}(67)(241)(1669)$ & \\
4021 & 91 & 226861496 & $(2)^{3}(28357687)$ & \\
4027 & -104 & 527257472 & $(2)^{3}\left(2^{2}\right)^{2}(7)(193)(3049)$ & \\
4051 & -56 & 551322528 & $(2)^{5}(3)(1933)(2971)$ & \\
4057 & -26 & 729226976 & $(2)^{5}(22788343)$ & \\
4093 & 127 & 320646728 & $(2)^{3}(40080841)$ & \\
4099 & 67 & 332880224 & $(2)^{5}(193)(53899)$ & \\
4111 & 109 & 226897784 & $(2)^{3}(28362223)$ & \\
4129 & -98 & 557796224 & $(2)^{3}\left(2^{2}\right)^{2}(19)(211)(1087)$ & \\
4153 & -122 & 558884768 & $(2)^{5}(13)(193)(6961)$ & \\
4159 & -83 & 234335448 & $(2)^{3}(3)^{2}(31)(67)(1567)$ & \\
4177 & 34 & 793463904 & $(2)^{5}(3)\left(3{ }^{2}\right)(918361)$ & \\
4201 & 127 & 267278408 & $(2)^{3}(103)(277)(1171)$ & \\
4219 & 1 & 288301664 & $(2)^{5}(7)(1287061)$ & \\
4231 & 7 & 294942552 & $(2)^{3}(3)(211)(58243)$ & \\
4243 & 85 & 286981544 & $(2)^{3}(35872693)$ & \\
4261 & 13 & 287072096 & $(2)^{5}(79)(113557)$ & \\
4273 & -53 & 251825912 & $(2)^{3}(13)(2421403)$ & \\
\end{tabular}


TABle 1. (continued)

\begin{tabular}{|c|c|c|c|c|}
\hline$p$ & $A$ & $\# K_{2} \mathcal{O}_{F}$ & $K_{2} \mathcal{O}_{F}$ & $C l\left(\mathcal{O}_{F}\right)$ \\
\hline $4 \overline{4297}$ & -131 & 258540416 & $\overline{(2)^{3}\left(2^{2}\right)^{2}(2019847)}$ & 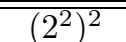 \\
\hline 4327 & 55 & 308430968 & $(2)^{3}(38553871)$ & \\
\hline 4339 & -128 & 872818656 & $(2)^{5}(3)(19)(151)(3169$ & (7) \\
\hline 4357 & -119 & 300064128 & $(2)^{3}\left(2^{2}\right)^{2}(3)(7)(13)(31)(277)$ & $(2)^{2}$ \\
\hline 4363 & 127 & 293035496 & $(2)^{3}(13)(2817649)$ & \\
\hline 4423 & -68 & 678133376 & $(2)^{3}\left(2^{2}\right)^{2}(5297917)$ & \\
\hline 4441 & -59 & 291978248 & $(2)^{3}(37)(241)(4093)$ & \\
\hline 4447 & 115 & 327955992 & $(2)^{3}(3)(7)\left(13^{2}\right)(11551)$ & \\
\hline 4483 & 133 & 458846808 & $(2)^{3}(3)(7)(19)(43)(3343)$ & \\
\hline 4507 & 91 & 323606792 & $(2)^{3}(1693)(23893)$ & \\
\hline 4513 & -50 & 1127785568 & $(2)^{5}(7)^{2}(13)(61)(907)$ & \\
\hline 4519 & 124 & 789244128 & $(2)^{5}(3)\left(3^{2}\right)(31)(79)(373)$ & \\
\hline 4549 & -86 & 810085472 & $(2)^{5}(7)(3616453)$ & \\
\hline 4561 & 37 & 346779776 & $(2)^{3}\left(2^{2}\right)^{2}(7)(387031)$ & $(2)^{2}$ \\
\hline 4567 & 4 & 735532544 & $(2)^{3}\left(2^{3}\right)^{2}(43)(33409)$ & $(2)^{2}$ \\
\hline 4591 & 133 & 375756264 & $(2)^{3}\left(3^{2}\right)(13)(157)(2557)$ & \\
\hline 4597 & -134 & 747504224 & $(2)^{5}(2551)(9157)$ & \\
\hline 4603 & 103 & 318625496 & $(2)^{3}(7)(5689741)$ & \\
\hline 4621 & -131 & 342124616 & $(2)^{3}(42765577)$ & \\
\hline 4639 & -41 & 368370176 & $(2)^{3}\left(2^{3}\right)^{2}\left(\mathbf{1 9}^{\mathbf{2}}\right)(1993)$ & $(2)^{2}$ \\
\hline 4651 & 136 & 759974816 & $(2)^{5}(307)(77359)$ & \\
\hline 4657 & 130 & 871034528 & $(2)^{5}(7)(13)(31)(9649)$ & \\
\hline 4663 & -20 & 867009408 & $(2)^{3}\left(2^{2}\right)^{2}(3)(2257837)$ & \\
\hline 4723 & -125 & 391395992 & $(2)^{3}(13)(3763423)$ & \\
\hline 4729 & 58 & 1160693888 & $(2)^{3}\left(2^{2}\right)^{2}(19)(477259)$ & \\
\hline 4759 & 28 & 893525024 & $(2)^{5}(7)(229)(17419)$ & \\
\hline 4783 & 85 & 608581928 & $(2)^{3}(76072741)$ & (7) \\
\hline 4789 & 97 & 361591776 & $(2)^{5}\left(3^{\mathbf{5}}\right)\left(7^{2}\right)(13)(73)$ & $(2)^{2}$ \\
\hline 4801 & 121 & 374007776 & $(2)^{5}(109)(107227)$ & $(2)^{2}$ \\
\hline 4813 & -107 & 360943256 & $(2)^{3}(45117907)$ & \\
\hline 4831 & -71 & 366576728 & $(2)^{3}(7)\left(\mathbf{1 9}^{\mathbf{2}}\right)(18133)$ & \\
\hline 4861 & -137 & 417409800 & $(2)^{3}(3)(5)^{2}(757)(919)$ & \\
\hline 4903 & 73 & 389371256 & $(2)^{3}(19)(2561653)$ & \\
\hline 4909 & 94 & 1006854752 & $(2)^{5}(193)(163027)$ & \\
\hline 4933 & 7 & 586541592 & $(2)^{3}(3)^{2}(7)(13)(89521)$ & \\
\hline 4951 & -11 & 566435016 & $(2)^{3}(3)^{2}(7)(1123879)$ & \\
\hline 4957 & -89 & 594452264 & $(2)^{3}(7)(10615219)$ & \\
\hline 4969 & 133 & 603100536 & $(2)^{3}(3)\left(\mathbf{3 1}^{\mathbf{2}}\right)(79)(331)$ & \\
\hline 4987 & -101 & 408631704 & $(2)^{3}(3)(13)(1309717)$ & \\
\hline 4993 & -17 & 569508056 & $(2)^{3}(13)(5476039)$ & \\
\hline 4999 & 139 & 450022664 & $(2)^{3}\left(7^{2}\right)\left(13^{2}\right)(6793)$ & \\
\hline
\end{tabular}


TABLE 2.

\begin{tabular}{|r|c|c|c|c|c|c|c|}
\hline$p$ & $v_{7}$ & $d_{1}$ & $d_{2}$ & $h_{1}$ & $h_{2}$ & 7 -rank $K_{2} \mathcal{O}_{F}$ & $\left(K_{2} \mathcal{O}_{F}\right)_{7}$ \\
\hline \hline 103 & 2 & 1 & 0 & 0 & 0 & 1 & $\left(7^{2}\right)$ \\
379 & 2 & 0 & 1 & 0 & 0 & 1 & $\left(7^{2}\right)$ \\
877 & 3 & 1 & 1 & 0 & 0 & 2 & $(7)\left(7^{2}\right)$ \\
1567 & 2 & 1 & 1 & 0 & 0 & 2 & $(7)^{2}$ \\
1597 & 2 & 1 & 0 & 0 & 0 & 1 & $\left(7^{2}\right)$ \\
1741 & 2 & 1 & 0 & 0 & 0 & 1 & $\left(7^{2}\right)$ \\
1801 & 3 & 1 & 1 & 0 & 0 & 2 & $(7)\left(7^{2}\right)$ \\
2029 & 2 & 0 & 1 & 0 & 0 & 1 & $\left(7^{2}\right)$ \\
2131 & 2 & 1 & 1 & 0 & 0 & 2 & $(7)^{2}$ \\
2161 & 2 & 1 & 0 & 0 & 0 & 1 & $\left(7^{2}\right)$ \\
2593 & 2 & 0 & 1 & 0 & 0 & 1 & $\left(7^{2}\right)$ \\
2707 & 3 & 1 & 0 & 0 & 0 & 1 & $\left(7^{3}\right)$ \\
2731 & 2 & 0 & 1 & 0 & 0 & 1 & $\left(7^{2}\right)$ \\
3037 & 3 & 0 & 1 & 0 & 0 & 1 & $\left(7^{3}\right)$ \\
3313 & 2 & 0 & 0 & 0 & 1 & 1 & $\left(7^{2}\right)$ \\
3469 & 2 & 1 & 1 & 0 & 0 & 2 & $(7)^{2}$ \\
3631 & 2 & 1 & 1 & 0 & 0 & 2 & $(7)^{2}$ \\
3877 & 3 & 1 & 1 & 0 & 0 & 2 & $(7)\left(7^{2}\right)$ \\
4513 & 2 & 1 & 1 & $\leq 1$ & 0 & 2 & $(7)^{2}$ \\
4789 & 2 & 1 & 0 & 0 & 0 & 1 & $\left(7^{2}\right)$ \\
4999 & 2 & 0 & 1 & 0 & 0 & 1 & $\left(7^{2}\right)$ \\
\hline
\end{tabular}

TABLE 3.

\begin{tabular}{|r|c|c|c|c|c|}
\hline \multicolumn{1}{|c|}{$p$} & $v_{13}$ & $d_{1}$ & $d_{2}$ & 13-rank $K_{2} \mathcal{O}_{F}$ & $\left(K_{2} \mathcal{O}_{F}\right)_{13}$ \\
\hline \hline 163 & 2 & 1 & 0 & 1 & $\left(13^{2}\right)$ \\
379 & 2 & 1 & 1 & 2 & $(13)^{2}$ \\
487 & 2 & 1 & 1 & 2 & $(13)^{2}$ \\
757 & 2 & 1 & 0 & 1 & $\left(13^{2}\right)$ \\
1723 & 2 & 1 & 1 & 2 & $(13)^{2}$ \\
1867 & 2 & 0 & 1 & 1 & $\left(13^{2}\right)$ \\
2797 & 2 & 0 & 1 & 1 & $\left(13^{2}\right)$ \\
3673 & 2 & 1 & 0 & 1 & $\left(13^{2}\right)$ \\
4447 & 2 & 1 & 0 & 1 & $\left(13^{2}\right)$ \\
4999 & 2 & 1 & 0 & 1 & $\left(13^{2}\right)$ \\
\hline
\end{tabular}




\section{REFERENCES}

[Br1] J. Browkin, The functor $K_{2}$ for the ring of integers of a number field, Banach Center Publ. 9 (1982), 187-195. MR0738813 (85f:11084)

[Br2] , On the p-rank of the tame kernel of algebraic number fields, J. Reine Angew. Math. 432 (1992), 135-149. MR1184763 (93j:11077)

[CH] P.E. Conner, J. Hurrelbrink, On the 4-rank of the tame kernel of $\mathrm{K}_{2} \mathrm{O}$ in positive definite terms, J. Number Theory 88 (2) (2001), 263-282. MR1832007|(2002h:11120)

[Ge] M. Geijsberts, The tame kernel, computational aspects, Ph.D. Thesis, Catholic University of Nijmegen (1991).

[Gr] G. Gras, Remarks on $K_{2}$ of number fields, J. Number Theory 23 (3) (1986), 322-335. MR0846962 (87j:11124)

[Ha] H. Hasse, Arithmetische Bestimmung von Grundeinheit und Klassenzahl in zyklischen kubischen und biquadratischen Zahlkörper, Abh. Deutsch. Akad. Wiss. 2 (1948), 3-95, (= Mathematische Abhandlungen, H.W. Leopoldt, P. Roquette (eds.), Band III, pp. 289379, Berlin, de Gruyter, 1975). MF0033863 (11:503d)

[HK] J. Hurrelbrink, M. Kolster, Tame kernels under relative quadratic extensions and Hilbert symbols, J. Reine Angew. Math. 499 (1998), 145-188. MR1631116 (2000a:11169)

[IR] K. Ireland, M. Rosen, A Classical Introduction to Modern Number Theory, SpringerVerlag, 1982. MF 0661074 (83g:12001)

[Ke] $\mathrm{F}$. Keune, On the structure of the $K_{2}$ of the ring of integers in a number field, K-Theory 2 (5) (1989), 625-645. MF0999397 (90g:11162)

[KK] H.K. Kim, J.S. Kim, Evaluation of zeta function of the simplest cubic field at negative odd integers, Math. Comp. 71 (239) (2002), 1243-1262. MR1898754 (2003h:11143)

[Ko1] M. Kolster, The structure of 2-Sylow subgroup of $K_{2} \mathfrak{o}, I$, Comment. Math. Helvetici 61 (3) (1986), 376-388. MR0860130 (88a:11125)

[Ko2] The structure of 2-Sylow subgroup of $K_{2} \mathfrak{o}, I I$, K-Theory 1 (5) (1987), 467-479. MR0934452 (89h:11077)

[Ko3] - A relation between the 2-primary parts of the main conjecture and the Birch-Tate conjecture, Canad. Math. Bull. 32 (2) (1989), 248-251. MR1006753 (90k:11154)

[Ko4] $K_{2}$ of rings of algebraic integers, J. Number Theory 42 (1) (1992), 103-122. MR1176424 (93f:11089)

[KM] M. Kolster, A. Movahhedi, Galois co-descent for étale wild kernels and capitulation, Ann. Inst. Fourier, Grenoble 50 (1) (2000), 35-65. MR.1762337 (2001d:11115)

[Ma] F. Marko, On the existence of p-units and Minkowski units in totally real cyclic fields, Abh. Math. Sem. Univ. Hamburg 66 (1996), 89-111. MF 1418221 (97k:11151)

[MW] B. Mazur, A. Wiles, Class fields of abelian extensions of $\mathbb{Q}$, Invent. Math. 76 (1984), 179-330. MR0742853 (85m:11069)

[Q1] H. Qin, Computation of $K_{2} \mathbb{Z}[\sqrt{-6}]$, J. Pure Appl. Algebra 96 (1994), 133-146. MR1303542 (95i:11135)

[Q2] - The 2-Sylow subgroups of the tame kernel of imaginary quadratic fields, Acta Arith. 69 (1995), 153-169. MR1316704 (96a:11132)

[Q3] - The 4-rank of $\mathrm{K}_{2} \mathrm{O}_{F}$ for real quadratic fields F, Acta Arith. 72 (1995), 323-333. MR1348200 (96f:11151)

[Q4] , Tame kernels and Tate kernels of quadratic number fields, J. Reine Angew. Math. 530 (2001), 105-144. MR1807269 (2002g:11167)

[ST] J.H. Silverman, J. Tate, Rational Points on Elliptic Curves, Springer-Verlag, New York, 1992. MR1171452 (93g:11003)

[Ta] J. Tate, Relations between $K_{2}$ and Galois cohomology, Invent. Math. 36 (1976), 257-274. MR0429837 (55:2847)

[Wa] L.C. Washington, Introduction to Cyclotomic Fields, Springer-Verlag, 1982. MR0718674 (85g:11001)

[Wi] A. Wiles, The Iwasawa conjecture for totally real fields, Ann. of Math. (2) 131 (1990), 493-540. MR,1053488 (91i:11163)

Institute of Mathematics, University of Warsaw, ul. Banacha 2, PL-02-097 Warsaw, Poland

E-mail address: bro@mimuw.edu.pl 